This document is the accepted manuscript version of the following article:

Hengsberger, M., Leuenberger, D., Schuler, A., Roth, S., \& Muntwiler, M. (2020). Dynamics of excited interlayer states in hexagonal boron nitride monolayers. Journal of Physics D: Applied Physics, 53(20), 203001 (28 pp.). https: //doi .org/10.1088/1361-6463/ab70c6

\title{
Dynamics of excited interface states in hexagonal boron nitride monolayers
}

\author{
M. Hengsberger ${ }^{1}$, D. Leuenberger ${ }^{1}$, A. Schuler ${ }^{1}$, S. Roth $^{2}$, M. \\ Muntwiler ${ }^{3}$ \\ ${ }^{1}$ Physik-Institut, Universität Zürich, Winterthurerstrasse 190, 8057 Zürich, \\ Switzerland \\ ${ }^{2}$ Laboratoire de Spectroscopie Électronique, Institut de Physique, École \\ Polytechnique Fédérale de Lausanne, 1015 Lausanne, Switzerland \\ ${ }^{3}$ Swiss Light Source, Paul-Scherrer-Institut, 5232 Villigen, Switzerland \\ E-mail: matthias.hengsberger@physik.uzh.ch
}

17 February 2020

\begin{abstract}
Hexagonal boron nitride $(h-\mathrm{BN})$ is the isoelectronic but insulating counterpart of graphene. Like graphene it can easily been grown as high quality nanotubes or as single layers on metal surfaces. Both materials can be exfoliated or transferred after single-layer growth from suitable substrates onto new surfaces. In view of electronic devices or optical sensors, for instance, the carrier dynamics in the conduction bands determine the device properties. The band edge of the unoccupied bandstructure of $h-\mathrm{BN}$ is dominated by two kinds of states, free-electron-like interlayer or interface states and a flat conduction band valley derived from $\pi^{\star}$-states. The measurement of the excited states and excited state lifetimes in $h-\mathrm{BN}$ is the main topic of the present article with a special focus on the dynamics close to the $\Gamma$-point. While the $\pi^{\star}$ conduction band minimum is strongly localised at the boron sites, the charge density of the free-electron-like states is outside the planes and is likely to be important for interactions like charge transfer with adjacent layers and substrates. We will review previous efforts to determine the nature of the bandgap and the bandstructure of unoccupied states with particular emphasis on but not restricted to single-layer $h-\mathrm{BN}$ epitaxially grown on a $\mathrm{Ni}(111)$ surface.
\end{abstract}

\section{Introduction: electronic structure of bulk and single-layer $h-\mathrm{BN}$}

In the past decade, pushed by the advent and technological advances of $2 \mathrm{D}$ materials starting with graphene [1,2], other elemental 2D single-layer systems [3], or singlelayer transition metal dichalcogenides [4-6], important research efforts were deployed in the quest for heterostructures with $2 \mathrm{D}$ dielectric materials or systems with defined conduction band valleys, the occupation of which could be used for information encoding $[7,8]$. Hexagonal boron nitride $(h-\mathrm{BN})$ is a wide band gap material that has a rather simple crystal structure and electronic configuration. Both, its use as bulk material, thin film, or even single layers bares great technological potential, and, while working 
almost exclusively with single layers, in this work we will try to build a bridge to bulk material as often as possible.

Beside for its electronic and optical properties, mechanical properties of single-layer membranes like stiffness, chemical inertness, and the possibility to grow nanotubes [9] or create nanoholes [10] have raised a lot of interest. The suitability of $h-\mathrm{BN}$ in important applications like nanofiltration [9] for osmosis or water desalination, as support material for (ordered) functional groups [11-13], functional coatings [14] and the like were demonstrated (for a general review about the use of atomically thin membranes, see Ref. [15]). Moreover, $h-\mathrm{BN}$ can be produced in high quality e.g.by chemical vapour deposition (CDV) on large-scale wafer supports and transferred by electro-chemical delamination [16], or as thin films by molecular beam epitaxy [17]. In electronic devices it is a suitable material for tunnelling barriers [18, 19] or dielectric spacers in transistors [20, 21]. Tunnelling barriers in graphene stacks with transmission depending on the angle of incidence were predicted from theoretical calculations [22, 23]. Finally mechanical and/or chemically protective encapsulation of 2D heterostructures was demonstrated in several papers [24-26].

Yet, in spite of numerous studies over several decades, there is still considerable controversy about details of its electronic structure and in particular the nature of the energy gap for bulk and single-layer $h-\mathrm{BN}$ [27-33]. This is important because $h-\mathrm{BN}$ used in p-n-junctions could be an important material for light emission or detection in the deep ultraviolet range due to the large bandgap of about $6 \mathrm{eV}[34,35]$. On the same footings, exciton dynamics in bulk $h$-BN became a field of intense research because of the high cathodo- and photoluminescence yields observed from bulk $h-\mathrm{BN}$ $[29,33,34,36,37]$. The high quantum efficiencies of up to $40 \%$ (see Fig. 1) and the observation of lasing in the deep ultraviolet under illumination with high-energy electrons [29] are reminiscent of direct band gap materials in contrast to bandstructure calculations for $h-\mathrm{BN}$, which mostly predict an indirect band gap [27, 28]. The direct band gap hypothesis was corroborated by the missing temperature dependence of the luminescence spectra [29]. Another indication is given by the lifetimes of intrinsic excitons: for diamond, which has an indirect bandgap of similar size than $h$-BN, the low luminescence efficiency comes along with very long exciton lifetimes of the order of hundreds of nanoseconds [38]. In contrast to this, Watanabe et al. reported lifetimes of about 600 ps for intrinsic excitons and long lifetimes of about 5 ns of excitons trapped at defects [39], thus of the same order of magnitude than typical exciton recombination times of $170-430$ ps of free excitons in $\mathrm{ZnO}$ [40]. On the other hand, the exciton spectra observed in luminescence spectroscopy reveal a fine structure which can directly be related to phonon-mediated exciton decay with phonons of correct energy and momentum giving evidence for an indirect band gap [33]. Thus, for the explanation of the high luminescence yields, excitons are expected to be the key, but the dynamics and the highly efficient direct or phonon-mediated recombination channels are not yet fully understood $[29,30,33,41]$. For a more detailed discussion, the reader is referred to recent papers in which these questions are reviewed [37, 42]. 
(a)
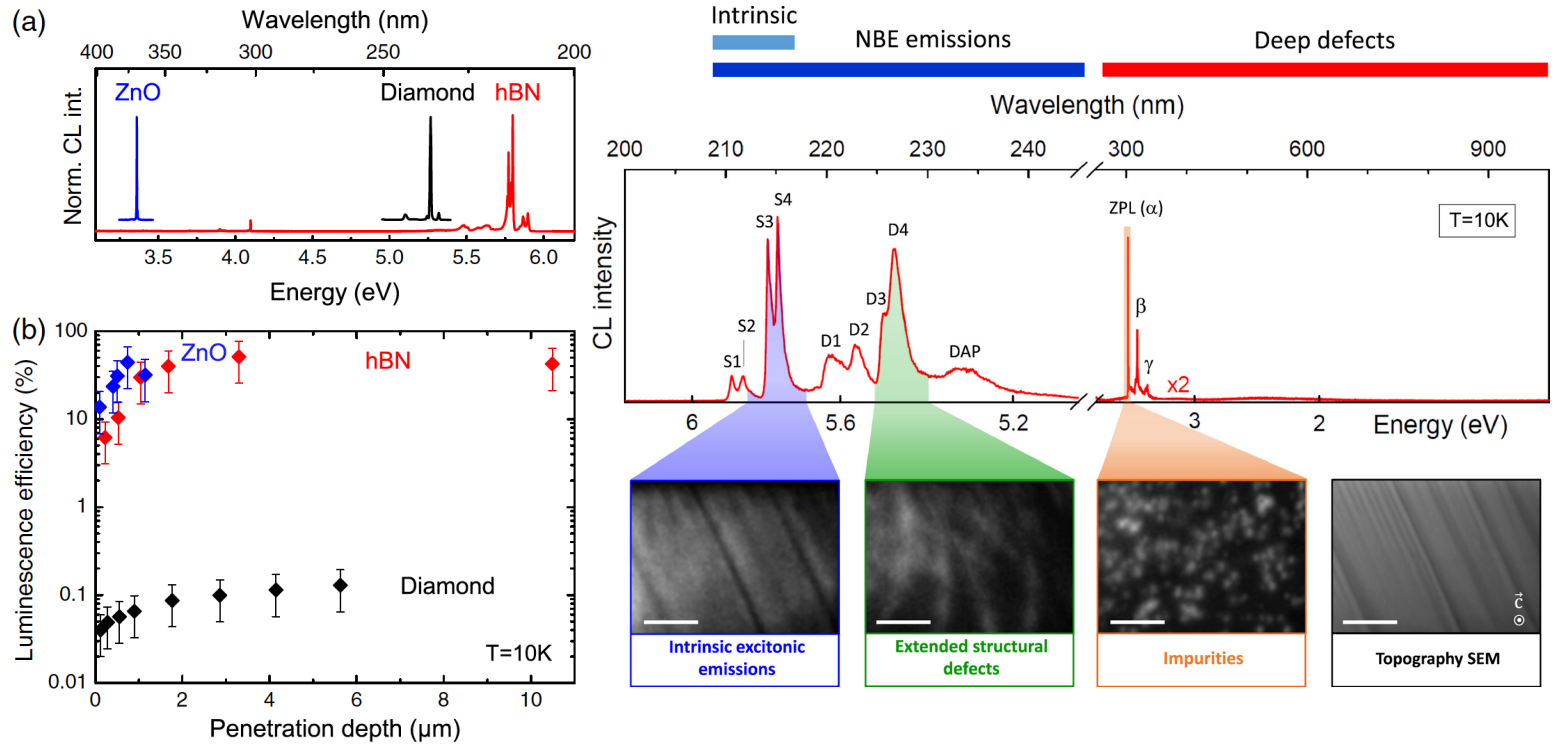

Figure 1. Cathodoluminescence intensity from $h$-BN. Left panel: Spectra and efficiencies as function of electron penetration depth for $\mathrm{ZnO}$ (direct bandgap), diamond (indirect bandgap) and $h$-BN. The efficiency is defined as generation rate of excitons divided by photon emission rate. Reprinted with permission from Ref. [37]. (Copyright 2019 by the American Physical Society). Right panel: Luminescence spectra and images of a $h-\mathrm{BN}$ single crystal structured with a focused ion beam. The spectral bands used for imaging are indicated: The S-series is associated with intrinsic luminescence due to exciton recombination in pristine $h-\mathrm{BN}$, the D-series represents emission from defects; the ZPL emission is associated with substitutional defects (carbon, oxygen) [43]. From Ref. [43] (Copyright 2019 by Institute of Physics Publishing).

Besides the large bandgap $h-\mathrm{BN}$ possesses the same structure and almost same lattice constant as and is iso-electronic to graphene. The growth of heterostacks of graphene and $h-\mathrm{BN}$ layers is not a trivial task but can be done e.g. by direct epitaxial growth on suitable substrates or by exfoliation and stacking in a chosen sequence and relative orientation [19, 44-46]. Lateral heterostructures of doped graphene and $h-$ $\mathrm{BN}$ were demonstrated. It was reported that carrier mobilities in graphene increase significantly in presence of a well-ordered $h$-BN layer [47-49]. Furthermore, $h-\mathrm{BN}$ constitutes a good dielectric tunnelling barrier between graphene layers depending on the relative structural orientation of the lattices [18, 19] even improving significantly the dissipation of heat produced in the source-drain channel through the dielectric layer to the gold gate contact [50]. Again, details of the interaction and the charge transfer between $h-\mathrm{BN}$ and graphene layers are still to be elucidated. A recent in-depth review concerning devices based on $h-\mathrm{BN} /$ graphene structures and the properties thereof can be found in Ref. [51].

Angle-resolved photoelectron spectroscopy (ARPES) is the prime tool to study the band structure, excitations, many-body effects, and finally dynamics in solid surfaces 
with full momentum and, if required, spin resolution [52]. The inherent surface sensitivity, often considered to be a drawback of this method, turns even into an advantage when investigating 2D systems like adsorbate layers on surfaces, surface states, or layered materials. Therefore, it was successfully employed to study monolayers and thin films of $h-\mathrm{BN}$ on all kinds of surfaces since the pioneering work of the group of Oshima in the 90s [53-55]. During the past decades, h-BN was studied as single-layers on a large variety of different transition metals [56-59], alloys [60, 61], and even insulating substrates like germanium [62] or sapphire [63]. On many surfaces, the lattice mismatch between h-BN and substrate is not negligible leading to surface reconstructions with large unit cells, the probably most famous being the so-called nanomesh [64-66]. A comprehensive overview on physical and chemical properties of $h$-BN-metal surfaces including many details about the preparation of single-layer $h-\mathrm{BN}$ can be found in a recent review [67], an introduction into the physics of $h-\mathrm{BN}$ and graphene monolayers and heterostructures in Refs. [68, 69]. We emphasise that the study of monolayer systems or thin films is important in view of applications because of differences between bulk and monolayer systems in terms of phonon spectra [55, 70] or exciton dynamics [41, 71], which must be reflected in the electronic structure as well. Of course, excited state lifetimes are expected to be significantly shorter for the monolayer system than for highquality bulk crystals. Even for essentially defect-free epitaxial layers like on $\mathrm{Ni}(111)$, the metal substrate provides a huge bath of low-energy electron-pair and phonon excitations which can be used to dissipate the excess energy. Moreover, surfaces or interfaces often offer a high defect density and, thereby, recombinations centres [72].

In this work we will review theoretical predictions and experimental findings on the unoccupied bandstructure of monolayer $h-\mathrm{BN}$ on metal substrates, mainly nickel. A particular emphasis is on the unoccupied free-electron-like interface states predicted to exist in bulk and single-layer $h-\mathrm{BN}[27,28,73]$. ARPES and, in particular time-resolved two-photon photoemission, are the methods of choice. As far as possible, the results will be compared to measurements of bulk $h-\mathrm{BN}$ and theoretical calculations. Note that we will try to distinguish between single, isolated or free-standing layers on one side, referred to as single layer or isolated layers, and monolayers of $h$-BN adsorbed on a solid substrate. The latter will be referred to as monolayers.

The paper is organised as follows: after a short introduction to the experimental methods and setups, and the sample preparation (Section 2 and Section 4) used in our studies, we will discuss the overall bandstructure and compare it to ARPES and inverse photoemission measurements (Section 5). Fitting the theoretical bands to ARPES data will allow us to establish the quasi-particle bandstructure and to estimate the quasiparticle energy gaps for $h-\mathrm{BN} / \mathrm{Ni}(111)$. The second half of the paper will be devoted to the dynamics in the conduction bands and excitons related to nearly-free-electron states present in the metal- $h-\mathrm{BN}$ interface (Section 5.3), and to magnetic properties and dynamics for the case of $h-\mathrm{BN} / \mathrm{Ni}(111)$ (Section 7 ). We will conclude with an outlook on $h-\mathrm{BN} /$ graphene heterostructures (Section 8). 


\section{Experimental methods}

Monolayers of $h$-BNgrown on various surfaces have bee studied extensively over the past years using all kinds of surface science techniques, in particular angle-resolved photoelectron spectroscopy (ARPES), scanning tunnelling microscopy, and low-energyelectron diffraction, as well as synchrotron based techniques like x-ray absorption spectroscopy. In this work, we will focus on photoelectron spectroscopies. The relevant techniques will be briefly described in this section; results from other techniques like $\mathrm{x}$-ray absorption will be mentioned but the reader interested in learning more about these techniques is referred to the cited publications.

\subsection{Angle-resolved photoelectron and inverse photoemission spectroscopy (ARPES and ARIPES)}

In purely two-dimensional systems like monolayer $h-\mathrm{BN}$, the $2 \mathrm{D}$ in-plane momentum $\hbar \overrightarrow{k_{\|}}$of the electrons is the most relevant quantum number to describe the dispersing electronic states. For the sake of simplicity the prefactor $\hbar$ will be omitted in the text, and the wavevector $\vec{k}$ will be referred to as electron momentum. In an ARPES experiment, electrons emitted due to the photoelectric effect after absorption of an ultraviolet photon, are detected in vacuum according to their kinetic energy $E_{\text {kin }}$ and emission direction. This allows the full momentum vector to be calculated in the coordinate system of the sample surface. Due to translational invariance of the crystalvacuum interface parallel to the surface plane, the projection of the momentum vector

onto the surface plane, $\overrightarrow{k_{\|}}$, is conserved in the emission process modulo a reciprocal lattice vector of the surface (see e.g. the book of Hüfner for a thorough discussion of the refraction of the photoelectron at the surface [52]). The energy of the state from which the electron is emitted can be calculated from the kinetic energy $E_{\text {kin }}$ in vacuum, the photon energy $h \nu$, and the workfunction $\phi$ of the surface. The energy will be given as $E-E_{F}$, always referenced to the Fermi level $E_{F}$ of the metal substrate. The binding energy scale often used in literature uses the same reference energy but with opposite sign. We will avoid using this binding energy scale in order to be consistent throughout this manuscript.

Thus, the full 2D initial state dispersion can be obtained from the experiment by means of the following two relations:

$$
\begin{aligned}
E-E_{F} & =E_{\text {kin }}+\phi-h \nu \text { and } \\
k_{\|} & =\sqrt{\frac{2 m_{e}}{\hbar^{2}} E_{\text {kin }}} \sin (\Theta) \approx 0.5123 \AA^{-1} \sqrt{E_{\text {kin }} / 1 \mathrm{eV}} \sin (\Theta),
\end{aligned}
$$

where the angle $\Theta$ is the polar emission angle between the surface normal and the direction of the photoelectron momentum in vacuum.

In angle-resolved inverse photoemission (ARIPES) a free electron with defined momentum is impinging onto the surface with an angle $\Theta$ with respect to the surface normal. It is adsorbed in a high-energy state $\left\{k_{\|}, E_{\text {kin }}\right\}$. It may then decay into a low- 
energy state at the same momentum under emission of a photon. The photon can then by detected in a spectrometer (spectrograph mode) or else at fixed photon energy $h \nu$ by a counting detector (so-called isochromat mode) [74]. The energy and momentum of the final state can then be calculated using the very same equations Eqn. 1 and Eqn. 2 given above [75]. Moreover, using phase-space arguments one can show that the ratio of total cross-sections or count rates of inverse photoemission and conventional (forward) photoemission scales as the squared ratio of the wavelength of the emitted photon in $\operatorname{ARIPES}(\approx 100 \mathrm{~nm})$ and photoelectron wavelength $(\approx 10 \AA)$ and is of the order of $10^{-4}$ [76]. This is partially compensated by using high electron currents of the order of hundreds of micro-amperes, but the photon counters have very low efficiencies of typically $\leq 10^{5}$ events/C, which leads finally to count rates of the order of $10 \mathrm{~Hz}[74]$. Moreover, using a beam of spin-polarised electrons, the spin character of the states can be studied and/or the exchange splitting of bands well below the energy resolution of the experiment [77]. All of the spectroscopies used in this work are sensitive to the last few atomic surface layers only owing the inelastic mean free path of electrons in solids, which is in the range of $1 \mathrm{~nm}$ for AR(I)PES in the low-energy range between $1 \mathrm{eV}$ and $100 \mathrm{eV}[52]$.

\subsection{Time-resolved two-photon photoemission (2PPE)}

Angle-resolved two-photon photoemission (2PPE) is very similar to conventional ARPES with the difference that intermediate states contribute to the spectral intensity [78]. The momenta and energies can be determined in the same way as described above once the peaks in the spectra can be assigned to initial or intermediate states in the two-photon process [79]. In addition, 2PPE gives spectroscopic access to unoccupied states with momentum resolution. As can be deduced from Eqn. 2, the momentum resolution depends on the kinetic energy of the photoelectrons. For low photon energies as often encountered in 2PPE experiments the momentum resolution is thus very high with the drawback that the momentum range accessible is small with respect to the dimensions of typical Brillouin zones. The energy resolution depends on the linewidth of the light $(\sim 50 \mathrm{meV})$ and usually is comparable to conventional photoemission and, thereby, superior to that of inverse photoemission (about $400 \mathrm{meV}$ [74]).

Moreover, the two-photon process relies on sequential absorption of two photons, and the signal intensity depends on the timing of the photons and the intrinsic dynamics and timescales of the excited states, as sketched in Fig. 2 [80]. The first photon usually is called the pump photon, the second photon ionizing the intermediate state $|k\rangle$ is called the probe photon. The timing can be controlled very precisely by controlling the length of the optical path. The 2PPE intensity is then recorded as function of time delay between the two light pulses, and delay zero refers to the situation when both light pulses arrive at the same moment on the sample. The width of the cross-correlation curve of the light pulses directly yields the temporal resolution of the experiment[81]. If the $2 \mathrm{PPE}$ signal decreases exponentially $I_{2 \mathrm{PPE}} \propto \exp (-\Delta t / \tau)$ with increasing time 

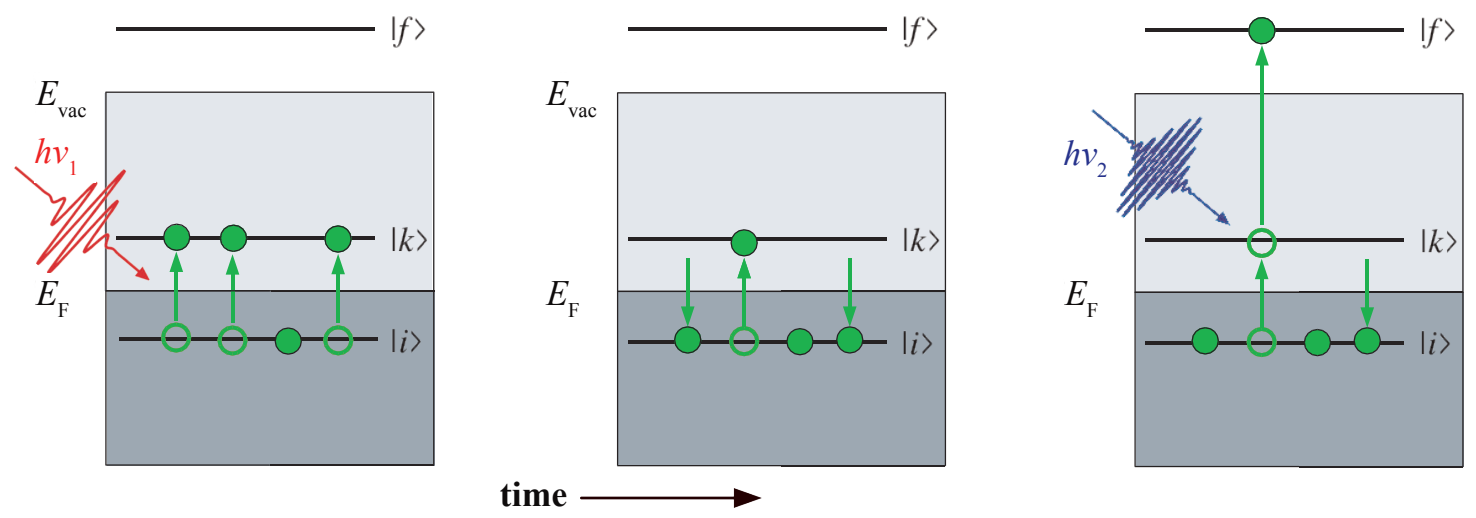

Figure 2. Population dynamics in two-photon photoemission (2PPE): The photoelectron is emitted after absorption of two photons with energies $h \nu_{1}$ and $h \nu_{2}$. Inbetween the two absorption events the excited state may relax. The signal recorded will, therefore, depend on the cross-correlation function of the light pulses and the lifetime of the intermediate state $|k\rangle$.

delay $\Delta t$ after absorption of the pump pulse, then the excited state dynamics can be described by a simple population decay with time constant (lifetime) $\tau$ or decay rate $\tau^{-1}$ (see e.g. the review of Bauer and Aeschlimann [82] for a detailed discussion). It is important to note that, in case of resonant excitation into an intermediate state, the energy of this state determines the peak position in the final $2 \mathrm{PPE}$ spectrum, if the initial state and the final free-electron-like state can both be considered continuum states in a first approximation $[79,83]$. This can be verified experimentally by checking the dependence of the peak position on photon energy [78], as the peak should move linearly with the variation of the probe photon energy.

We emphasise that in case of an exciton, the first optical transition of the $2 \mathrm{PPE}$ process measures the energy of the exciton state in contrast to inverse photoemission, in which an electron is added to the system. For the latter the peak position corresponds to the final state of the inverse photoemission process and, thereby, to the energy of the quasiparticle state.

Finally, by comparing the spectral linewidth of the 2PPE peaks with the lifetime of the same state as measured in 2PPE, one realises that the peak widths are dominated by additional broadening rather than purely limited by the inverse lifetime. This was pointed out very early by Bokor [84] and later on described theoretically by using optical Bloch equations, i.e. a set of coupled differential equations derived from the Liouvillevan Neumann density matrix formalism [81, 85, 86]. Briefly, these equations contain, beside the lifetime which characterises the population decay, a dephasing time which characterises the loss of coherence in the excited state. As a consequence, the excited intermediate state wavepacket broadens and decays with a rate which is given by the sum of dephasing and population decay rates. In solid surfaces, the linewidth is mostly determined by dephasing rather than the decay rate of the population of the excited state. 


\section{Experimental setup for (time-resolved) photoelectron spectroscopy}

Most of our experiments were carried out in our home laboratories. Every experimental station is equipped with a He discharge source for conventional valence band spectroscopy. The samples are mounted on 5-axes goniometers which allow all emission angles to be reached by rotation of the sample in front of the analyser. The photoelectrons are detected in commercial hemispherical electrostatic analysers [87-90]. The energy and angular resolution were typically set to $50 \mathrm{meV}$ and $\pm 1^{\circ}$, respectively. Most time-resolved spectra shown were taken with a bias voltage of -3 to $-10 \mathrm{~V}$ applied between the sample and the analyser in order to avoid the low-transmission range of the analyser at very low kinetic energies. The bending of the photoelectron trajectories due to this bias field were corrected for using the algorithm described in Ref. [79]. Moreover, spin-resolved spectra can be taken at the COPHEE endstation of the Swiss Light Source [91]. The inverse photoemission data were taken at the University of Münster (Germany) by the group of M. Donath [92], and their experimental setup is described in detail elsewhere [93].

The femtosecond light pulses were produced by a commercial Ti:sapphire oscillator (Coherent Mira Seed) providing a $76 \mathrm{MHz}$ pulse train with $30 \mathrm{~nm}$ bandwidth at a centre wavelength tunable between 790 and $830 \mathrm{~nm}(1.57$ to $1.49 \mathrm{eV})$. The pulses were frequency-doubled in a $\beta$-barium borate (BBO) crystal. Two-photon photoemission was done using either single-colour frequency-doubled pulses or a two-colour setup with fundamental and frequency-doubled pulses [94]. If higher pulse energies or broadly tunable pulses are required, the seed pulses provided by the oscillator were amplified by means of a regenerative chirped pulse amplifier (Coherent RegA 9050). After re-compression, pulses have pulse energies of about 4-6 $\mathrm{\mu J}$ at a photon energy of $h \nu=1.55 \mathrm{eV}(800 \mathrm{~nm})$ and a repetition rate of 30 to $250 \mathrm{kHz}$. The spectral width and the pulse duration after the RegA typically were $58 \mathrm{meV}(30 \mathrm{~nm})$ and $45-50 \mathrm{fs}$, respectively, assuming gaussian pulse shapes [95, 96]. The pulses could be focused into BBO crystals in order to generate the second or forth harmonic, or else into an optical parametric amplifier (Coherent OPA9450) providing a wavelength tunable between $470 \mathrm{~nm}(2.64 \mathrm{eV})$ and $700 \mathrm{~nm}(1.77 \mathrm{eV})$ [97]. Time-resolved 2PPE measurements could be realised using almost all of these wavelength combinations. Typical fluences range between about $10 \mathrm{\mu J} / \mathrm{cm}^{2}$ for spectroscopy [94] and $1 \mathrm{~mJ} / \mathrm{cm}^{2}$ in the pump pulse for achieving high excitation densities $[89,95]$. The width of the pump-probe pulse crosscorrelation function of the experiment was always measured by $2 \mathrm{PPE}$ directly on a reference sample. Moreover, recently, the range of photon energies was extended by developing a new high-harmonic generation (HHG) setup. For measuring e.g. the $\pi^{\star}$-band at the $\mathrm{K}$-point of $h-\mathrm{BN} / \mathrm{Ni}(111)\left(k_{\|} \approx 1.7 \AA^{-1}\right)$, a photon energy of about $h \nu \geq 12.5 \mathrm{eV}$ is necessary. Thus, a photon energy of $15 \mathrm{eV}$ as provided by our own HHG setup [90, 98], should allow us the dynamics of unoccupied states at the border of the Brillouin zone (BZ) to be studied with momenta of the order of $1.5 \AA^{-1}$. 
a)

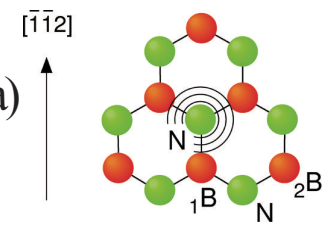

b)

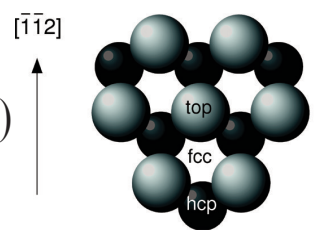

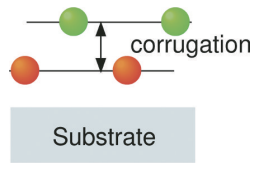

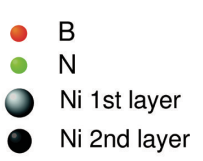

c)

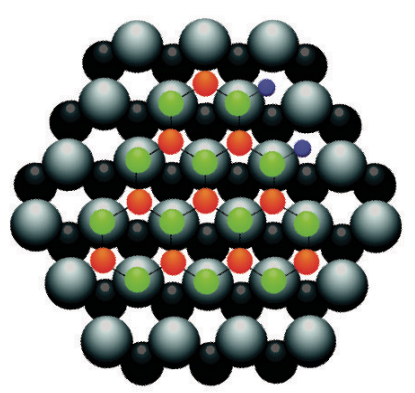

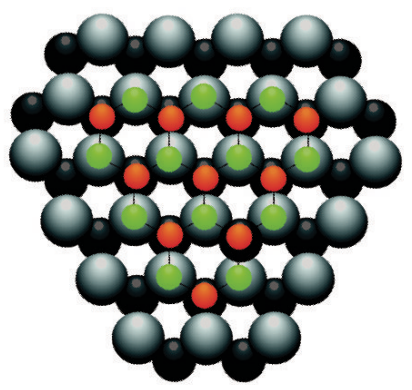

d)

Figure 3. Atomic structure of $h-\mathrm{BN} / \mathrm{Ni}(111)$. a) Structure of the hexagonal lattice (red boron, green nitrogen) with first and second nearest neighbours of the central nitrogen atom. b) Top, fcc, and hcp bonding positions on $\mathrm{Ni}(111)$. Inset and c) and d) Adsorption structures with lowest energies [102]: c) N on top of outmost Ni atom, B in fcc hollow site; d) $\mathrm{N}$ in top position, B in hcp hollow site. Reproduced from Ref. [103].

\section{Sample preparation and characterisation}

$h-\mathrm{BN}$ monolayers can conveniently be grown by chemical vapour deposition using borazine $\mathrm{H}_{3}(\mathrm{BN})_{3} \mathrm{H}_{3}$ as precursor on various metal surfaces [67, 68]. Depending on the lattice constants and surface atomic structure of the substrate, one obtains epitaxial growth [53, 99], moiré structures [57], or mesh-like, strongly corrugated films $[13,58,59,61,64,100,101]$.

Most measurements presented in this work were taken from $h-\mathrm{BN} / \mathrm{Ni}(111)$. The layers grow in an epitaxial fashion with very little corrugation due to the similar lattice constants of $h-\mathrm{BN}$ and $\mathrm{Ni}(111)$ [104]. Possible adsorption structures and energies as shown in Fig. 3 were calculated $[102,105]$ and experimentally characterised by photoelectron diffraction [99, 104]: It turned out that the most favourable geometry corresponds to $\mathrm{N}$ atoms sitting on top of first layer $\mathrm{Ni}$ atoms while $\mathrm{B}$ occupies hollow sites corresponding to hcp stacking with respect to the second Ni layer. The corrugation is such that $\mathrm{N}$ atoms, which have a partial negative charge are slightly pushed away from the Ni surface due to a small negative surface charge owing to charge transfer from $h-\mathrm{BN}$ to the metal substrate [65].

The $h-\mathrm{BN}$ monolayers were grown on single crystalline $\mathrm{Ni}(111)$ as well as on $80 \mathrm{~nm}$ thick nickel films under ultrahigh vacuum conditions (base pressure of $10^{-10}$ mbar) by chemical vapour deposition of $\sim 100 \mathrm{~L}$ ( 1 Langmuir $=10^{-6}$ torr sec) of borazine while keeping the substrate surface at a temperature of $1072 \mathrm{~K}$ [53]. The quality of the $h$-BN monolayers was verified by ultraviolet and x-ray photoelectron spectroscopy and low energy electron diffraction. Moreover, the work function of the sample was constantly monitored during the measurements by recording the whole energy range of the $2 \mathrm{PPE}$ spectra. It slightly scattered within $\pm 0.05 \mathrm{eV}$ around $3.6 \mathrm{eV}$ for different preparations and nickel substrates. During the experiments no changes of the work function were 
observed, thus no optically induced desorption of the hexagonal boron nitride monolayer occurred under the intense pump pulse radiation. The $h-\mathrm{BN}$ monolayers remained stable at room temperature and at temperatures up to $900 \mathrm{~K}$ and thereby permitted long measurement periods under reproducible conditions. The sample temperature was controlled by means of pyrometer and a thermocouple. Unless otherwise stated all our measurements were taken at room temperature.

\section{Quasiparticle bandstructure of monolayer $h-\mathrm{BN}$}

Over several decades, many bandstructure calculations were published for $h-\mathrm{BN}$. Starting from simple tight-binding models [106] the models were refined by using more extended basis sets $[27,73]$ and many-body effects [28] in order to get more and more precise pictures of this seemingly simple system. Nowadays there is agreement about the fact that many-body effects must be included in order to obtain more realistic values for the size of the energy gap $[28,30]$. Van der Waals corrections were shown to be important for getting the correct adsorption geometries [105]. For the calculation of exciton binding energies, the use of the Bethe-Salpter ansatz proved to yield good results [30, 37, 107-109]. Despite all these efforts, however, the nature of the energy gap, i.e. direct vs. indirect, was heavily debated: For bulk $h-\mathrm{BN}$ it was claimed that perfect h-BN single crystals have a direct band gap of $5.971 \mathrm{eV}$ and exciton absorption bands with a maximum exciton binding energy of $0.149 \mathrm{eV}$ [29]. Theoretical work interpreted the same data in terms of an indirect gap of $5.95 \mathrm{eV}$ with the exciton absorption bands resulting from a direct gap of $6.47 \mathrm{eV}$, what eventually implies a much larger exciton binding energy of $0.72 \mathrm{eV}$ [30]. More recently, a careful analysis of one- and two-photon photoluminescence spectra gave strong evidence for a phonon-mediated exciton decay and thus for an indirect bandgap [33].

The situation is different for single-layer $h-\mathrm{BN}$. Here, the valence band maximum is found at $\overline{\mathrm{K}}$, while the lowest lying unoccupied state is a nearly-free-electron-like (NFE) state at $\bar{\Gamma}[28,102,109,110]$. Due to the weak oscillator strength for an optical transition from the VBM at $\overline{\mathrm{K}}$ to the NFE state at $\bar{\Gamma}$, the material is believed to behave as direct bandgap material with the direct $\pi-\pi^{\star}$ gap located at $\overline{\mathrm{K}}[109]$.

In this section we will review the overall bandstructure and discuss the $\sigma$-, and $\pi$ bands in a first place and then the so-called interlayer or NFE states. The calculations will be compared to experimental results in sections 5.2 and 5.4.

\subsection{Generic bandstructure of $s p^{2}$-bonded layers}

Typical $\mathrm{sp}^{2}$-bonded networks in honeycomb structures possess an apparently simple electronic structure, the basics of which can be captured already using the tight-binding approximation. This had first been realised by Wallace in 1947 who described the generic bandstructure and basic electronic properties of graphite and its single-layer form, known today as graphene [111]. For a more detailed description including the 

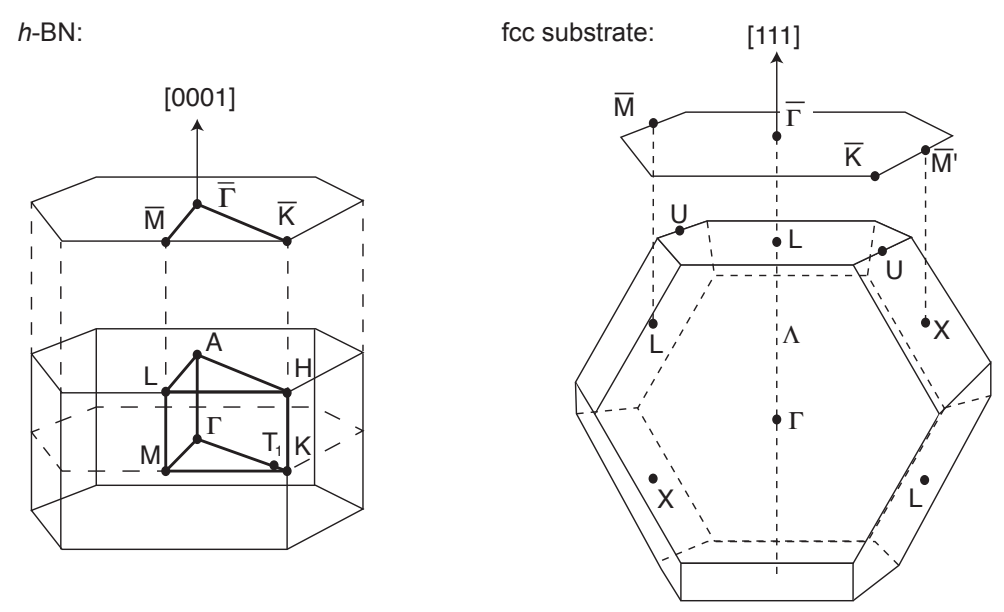

Figure 4. Brillouin zone of $h-\mathrm{BN}$. Left-hand-side: hexagonal bulk BZ of $h-\mathrm{BN}$ with high symmetry points and their projection onto the surface BZ of $h-\mathrm{BN}$. Right-handside: BZ of a fcc metal like nickel and the surface BZ which corresponds to the (111) surface.

case of graphene the reader is referred to the vast literature about these single-layer systems, as given e.g. in the review of Greber [69]. The description is general such that it can directly be extended to the case of $h-\mathrm{BN}$.

In networks of atoms with s- and p-like valence states, the electronic bands can be modelled using bands derived from molecular $\sigma$ and $\pi$ states. With one s-state and three p-states per atom, we obtain four combinations, each one twofold spin-degenerate. For a honeycomb lattice like graphene or single-layer $h-\mathrm{BN}$, every atom has three nearest neighbours. According to LCAO (linear combination of atomic orbitals) theory the cylindrical symmetry around a bond axis between two neighbouring atoms enforces $\sigma$ symmetry of the in-plane bonds between atoms. This means that every atom is bonded to the three neighbours by $\sigma$-bonds resulting from combinations of $2 \mathrm{~s}$ and $2 \mathrm{p}_{x}$ and $2 \mathrm{p}_{y}$ states, if $x$ and $y$ denote the coordinates in the plane, and $z$ the coordinate axis normal to the plane. The remaining $\mathrm{p}_{z}$-states are sticking out of the plane and hybridise to form the strongly dispersing $\pi$-bands with an occupied band width of about $8 \mathrm{eV}$ for $h$-BN [28]. No surface states were found for $h$-BN bulk in all-electron full-potential augmentedplane-wave calculations [73] suggesting that the electronic structure as observed in photoemission experiments can be expected to be representative of that of bulk $h-$ BN. The results of these calculations are displayed in Fig. 5 together with ARPES experiments from a bulk crystal [112] highlighting the excellent agreement between the theoretical and experimental bandstructure of bulk $h-\mathrm{BN}$.

The momenta of the valence and conduction band edges are of particular interest because they determine the nature of the band gap and, thereby, the probabilities of optical transitions in semi-conductors. In $h$-BN bulk crystals and single layers, the valence band maximum is found very close to (point $T_{1}$ along the line $\Gamma K$ ) and at $K$, respectively $[28,102,113]$. The nomenclature of the high-symmetry points in reciprocal 
a)

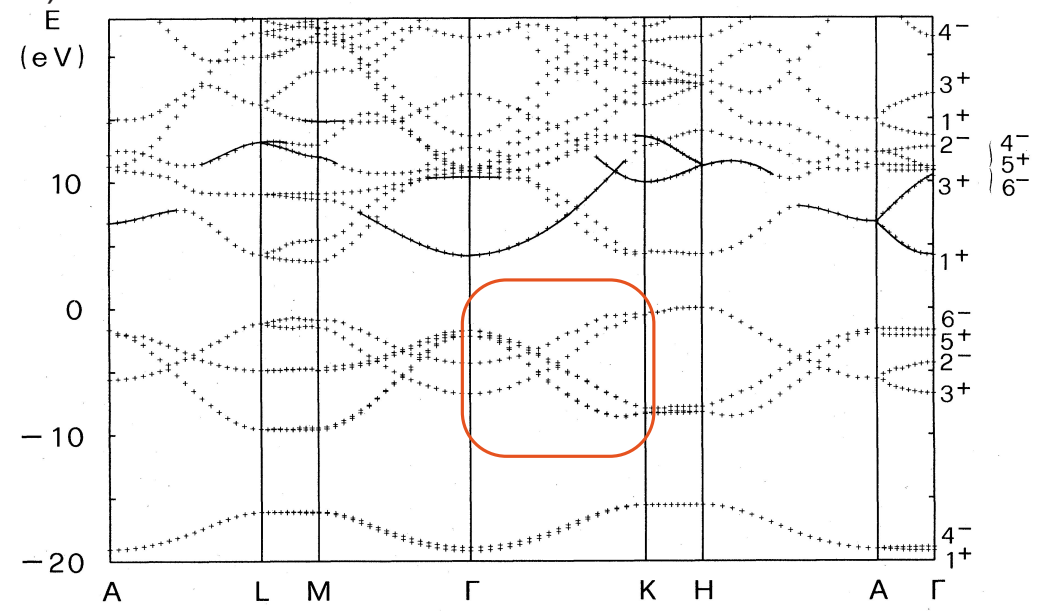

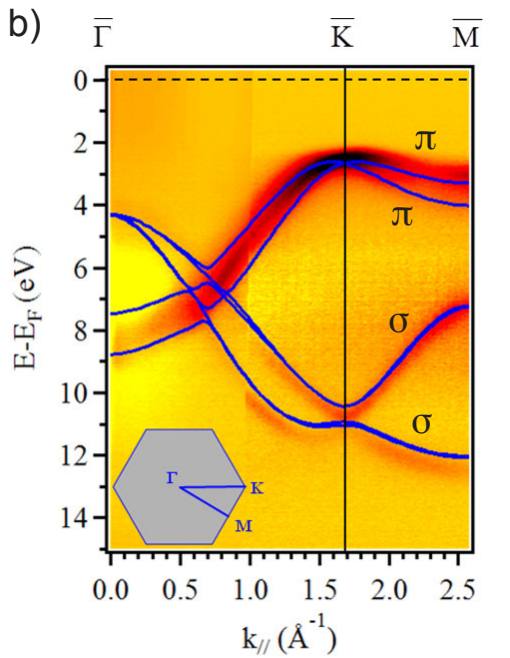

Figure 5. Bulk bandstructure: theory and experiment. a) Results of augmentedplane-wave calculations along high-symmetry directions in the bulk BZ. Reprinted with permission from Ref. [27]. (Copyright 1985 by the American Physical Society). The energy zero refers to the valence band maximum and the red rectangle indicates the range of the ARPES data shown in (b). b) ARPES data taken with $h \nu=100 \mathrm{eV}$ from $\sim 70 \mathrm{~nm}$ thick $h$-BN-films exfoliated from a commercial single crystal. Energy bands resulting from ab initio $\mathrm{G}_{0} \mathrm{~W}_{0}$ calculations are superimposed as blue lines. The labels $\sigma$ and $\pi$ were added by us. Reprinted with permission from Ref. [112]. (Copyright 2017 by the American Physical Society).

space is defined in Fig. 4. For bulk [112] and various single-layer systems (e.g. Refs. [53, 102], see Section 5.2) this was confirmed by ARPES, as shown in Fig. 5(b) for the case of a thick single-crystalline flake. According to calculations, the $\pi$ conduction band minimum is at $\mathrm{M}$ for bulk $h-\mathrm{BN}$, and the energy gap is of the order of $6 \mathrm{eV}$ with a large scatter between different calculations and functionals used for incorporating many-body interactions in the calculations [28, 108]. The conduction band minimum consists of anti-bonding $\pi^{\star}$ states, and the corresponding charge density is localised on the B-atoms. It is noted in passing that the self-energy corrections for the energies of the $\pi^{\star}$ band are strongly $k$-dependent and of the order of $0.6 \mathrm{eV}$ at $\mathrm{K}$ and $\mathrm{M}$ [28]. The same holds for the single layer (free-standing or adsorbed) with the difference that the $\pi^{\star}$ band bottom is found at $\mathrm{K}[28,102,109]$.

\subsection{Occupied states in monolayer $h-B N$}

A convenient and direct way to investigate the occupied bandstructure is given by ARPES. An example for bulk $h-\mathrm{BN}$ was already shown in Fig. 5(b). The literature for monolayer systems is much more abundant, on one hand because of technical issues in growing high-quality single crystals with suitable sizes [112], on the other hand because the combination of $h$-BN with various solid substrates of different lattice structure and lattice constants bares great potential for future technology employing 2D materials. 


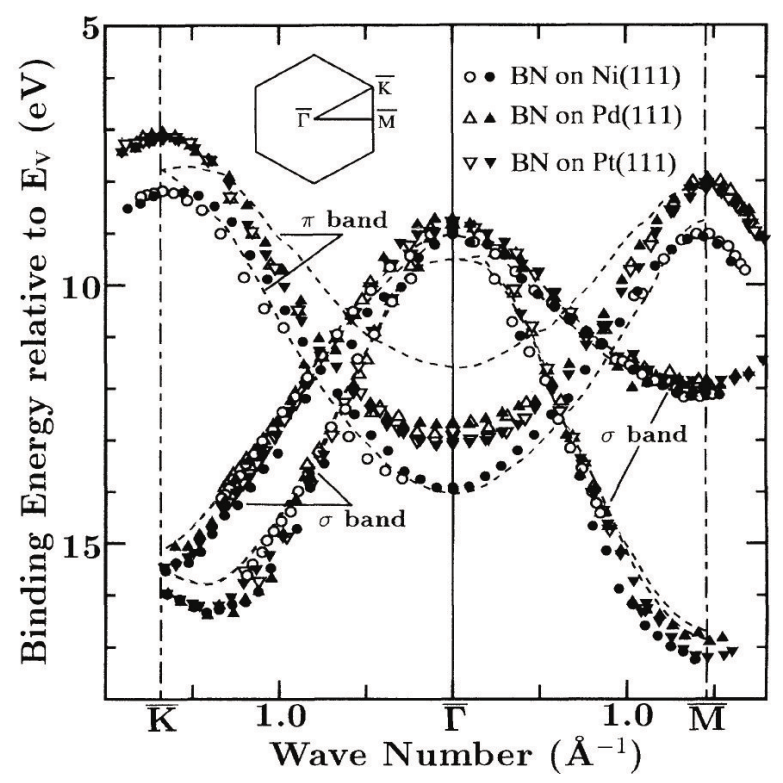

Figure 6. Dispersion of $\sigma$ - and $\pi$-bands from ARPES data for three surfaces and two different photon energies: $h-\mathrm{BN} / \mathrm{Ni}(111)$ (circles), $h-\mathrm{BN} / \mathrm{Pd}(111)$ (upward triangles) and $h-\mathrm{BN} / \mathrm{Pt}(111)$ (downward triangles); open and solid symbols refer to data taken with $\mathrm{HeI} \alpha-(21.2 \mathrm{eV})$ and $\mathrm{He} \mathrm{II} \alpha$ radiation $(40.8 \mathrm{eV})$, respectively. The data were aligned with the $\sigma$-band at $\bar{\Gamma}$ and are displayed against the energy with respect to $E_{\mathrm{vac}}$. Dashed lines are results from bandstructure calculations [73]. Reprinted with permission from Ref. [53] (copyright (1995) by the American Physical Society).

Moreover, several theoretical studies have been published, reproducing successfully the bandstructure and energy alignment, density of states (DOS), and adsorption structure to various levels of sophistication including recently van der Waals dispersion corrections for the computation of large $h-\mathrm{BN} /$ metal surface reconstructions [105].

In comparing photoemission data from monolayer $h-\mathrm{BN}$ on several metal surfaces, Nagashima, Oshima and co-workers realised in the 90s of the last century that the $\sigma$ and $\pi$ bands of $h-\mathrm{BN}$, as well as the 1s core levels of boron and nitrogen are close together in energy if the data were aligned with respect to the vacuum level $E_{\text {vac }}$ rather than to the Fermi level $E_{F}$, as can be seen in Fig. 6 [53]. This indicates that the layer on the substrates are physisorbed on the metal surfaces without forming strong covalent bonds [68].

As an exception, it can be seen that the $\pi$-band in $\mathrm{Ni}$ is lower in energy by $1 \mathrm{eV}$, what was interpreted as stronger binding on Ni surfaces on the kink to chemisorption [53]. The out-of-plane $\mathrm{p}_{z}$-states hybridise with $\mathrm{Ni}$ d-states and are lowered in energy, while the strong in-plane $\sigma$ bonds from $\mathrm{s}, \mathrm{p}_{x}$, and $\mathrm{p}_{y}$ orbitals are "floating" on the average potential in the film and align, therefore, to the vacuum level. This picture was corroborated several times by theory work $[65,102,110]$. 


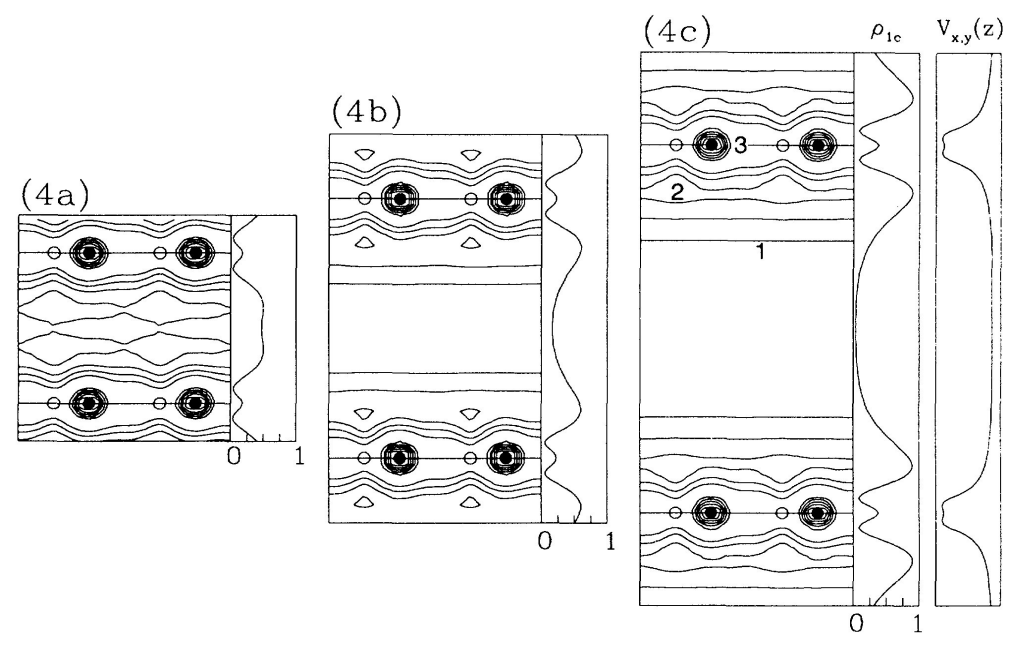

Figure 7. Charge density of the NFE interlayer states at $\bar{\Gamma}$ for three different values of layer separation $d$ : (4a) $d=5.5 \AA$, (4b) $d=9.5 \AA$, and (4c) $d=13.5 \AA$. We recall that the interlayer distance in bulk $h-\mathrm{BN}$ is $d=3.3 \AA$. Filled circles denote $\mathrm{N}$ atoms, open circles B atoms. The contours labeled 1, 2, and 3 correspond to charge densities of $0.95,2.905$, and $1.89 \times 10^{-4} \AA^{-3}$, respectively. Note that in these calculations, AA stacking (B atom over B atom) was assumed in contrast to AA' (B atom over $\mathrm{N}$ atom) stacking in bulk $h-$ BN. Reprinted with permission from Ref. [28]. (Copyright 1995 by the American Physical Society).

\subsection{Nearly-free-electron interlayer states in $h-B N$}

The overall bandstructure discussed in the preceding section can fairly well be reproduced in tight-binding calculations based on atomic-like s- and p-states [106]. An extension of the basis set, however, leads to the appearance of new states, which are very similar to those already predicted theoretically [114] for pristine and Li-intercalated graphite and found in inverse photoemission experiments [115].

Using augmented plane waves within the local-density full-potential method such new states indeed were found in the energy gap between bonding and anti-bonding $\sigma$ and $\pi$-states at $\Gamma$ for $h$-BN [27]. In contrast to the case of graphite, however, these states, which were termed interlayer states, do not arise by hybridisation of surface states. In thick slabs, Catellani and co-workers could show that the wavefunctions of the interlayer states converge to Bloch states and that no surface solution splits off [73]. The charge density of these states is mostly localised between single layers, as is shown in Fig. 7 for different layer distances. The first state is even under reflection at the atomic planes and belongs to the representation $\Gamma_{1}^{+}$using the notation of Robertson [106]. In the direction normal to the planes $(\Gamma \mathrm{A})$ the state disperses in a free-electron-like fashion, and the dispersion folds back at the border of the BZ. This leads to a second state at $\Gamma$, this time with odd symmetry $\Gamma_{3}^{-}$under reflection at the atomic planes [27]. As already pointed out by Catellani et al. there is substantial hybridisation of these interlayer states and $\pi$ - and $\sigma$-states with the same symmetry. This leads to significant contributions of 

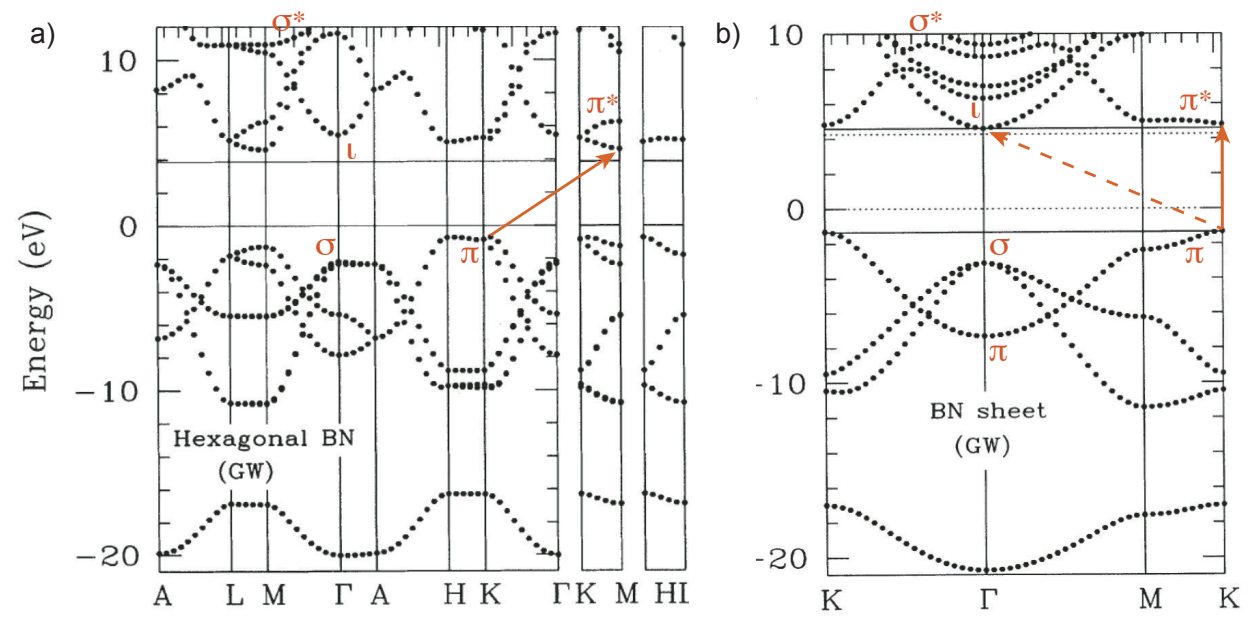

Figure 8. Bandstructures for bulk and singe-layer $h-\mathrm{BN}$. The eigenvalues were obtained by DFT in the Local Density Approximation. Many-body effects were included by screened Coulomb potentials within the GW approximation. The two calculations were displaced vertically such that the $\sigma$-bands at $\Gamma$ are aligned. Some bands are specified with red Greek letters and the optical transitions of lowest energy are indicated by red arrows. The zero of energy refers to the valence band maximum in the LDA calculation (not shown here). For the nomenclature of the high-symmetry points see Fig. 4. Reprinted with permission from Ref. [28] (Copyright 1995 by the American Physical Society); the red arrows and labels were added by us.

the nitrogen sites to the overall charge density of the interlayer states which amount to $13 \%$ and $33 \%$ for the lower $\Gamma_{1}^{+}$and the energetically higher $\Gamma_{3}^{-}$state, respectively [73]. The contribution of the boron sites can be neglected due to the lower electronegativity of boron. Thus, the interlayer states have a pronounced atomic character in the case of $h-\mathrm{BN}$, what was put forward as reason for the missing surface states in $h-\mathrm{BN}$ in contrast to the case of graphite.

For single-layer $h-\mathrm{BN}$, similar states are found in calculations for isolated layers $[28,109]$ and layers supported on metals [42, 102, 113]. For comparison, we plot in Fig. 8 the results for bulk and single-layer $h-\mathrm{BN}$ out of the same calculations:

The calculations were aligned at the binding energy of the $\sigma$-band at $\Gamma$. One can see that (i) the energy gap increases upon going from bulk to single layer, (ii) that the principal energy gap is indirect in both cases: The minimum of the bulk $\pi^{\star}$-band is found at the M-point of the bulk BZ but at $\mathrm{K}$ ( or $\overline{\mathrm{K}}$ ) for the isolated layer. The valence band maximum is given by the $\pi$-band in both cases and the maximum is found at $\mathrm{K}$ or close to $\mathrm{K}$ ( $\mathrm{T}_{1}$ point on the line $\left.\Gamma \mathrm{K}\right)$ for single-layer and bulk $h-\mathrm{BN}$, respectively [28]. For bulk $h-\mathrm{BN}$ state-of-the-art calculations predict the conduction band minimum to be at $\mathrm{M}[27,28,30]$. As a consequence, the excitation and recombination of $\pi-\pi^{\star}-$ excitons must involve a phonon and the signature of phonons with suitable energy and momentum indeed was found in luminescence spectra [33], providing strong evidence for an indirect bandgap.

For the isolated layer and with many-body corrections included, the interlayer state 


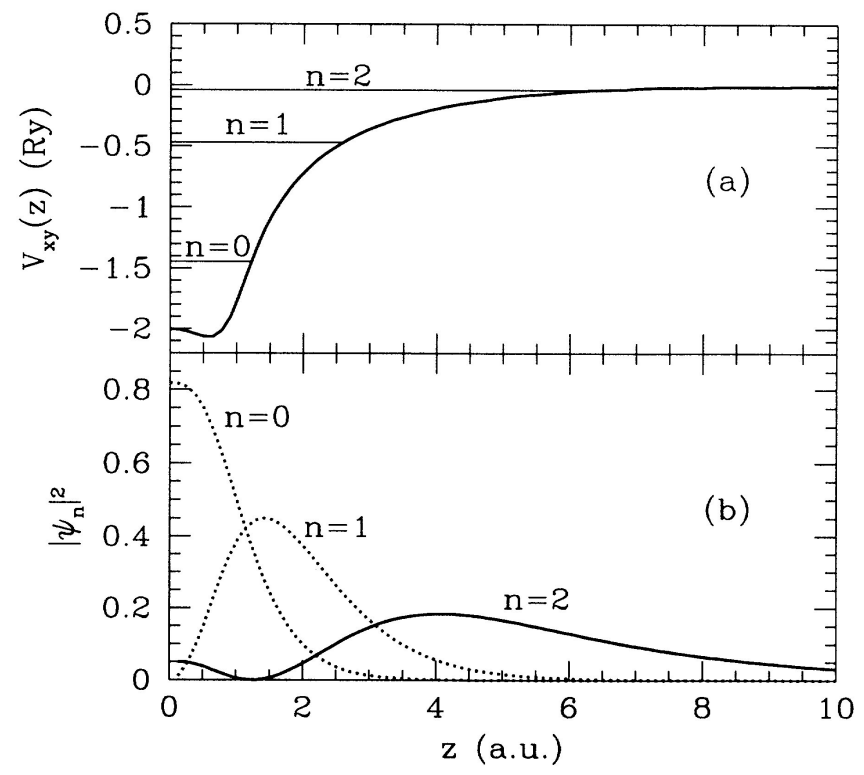

Figure 9. Interlayer bound states for an isolated sheet of $h-\mathrm{BN}$. (a) Average 1D potential of $h-\mathrm{BN}$ layers along the layer normal. The potential levels off at the vacuum level for large distance. The numbers $n$ are quantum numbers of bound states found by solving the 1D Schrödinger equation. (b) Charge density for the first three bound states. The curve for $n=2$ is close to the charge distribution of the interlayer state at $\Gamma$. Reprinted with permission from Ref. [28]. (Copyright 1995 by the American Physical Society).

becomes the lowest unoccupied state at $\bar{\Gamma}$. This was confirmed by calculations for adsorbed and isolated layers [102] and experiments [92] as we will see in the following. Thus, in this limit the gap is indirect as well. It was pointed out, however that the matrix element for this transition is negligible, probably due to the small real space overlap of the corresponding wavefunctions [109]: The authors suggest to treat the single layer like a direct bandgap material where the $\pi$ - $\pi^{\star}$-transition at $\mathrm{K}$ defines the optical gap.

There is another class of states similar to the interlayer states, which behave as free electrons parallel to the planes and are located outside the surface. They are bound by the Coulomb potential due to the electronic polarisation at the surface induced by their own charge. In (ideal) metals, the polarisation generates the same field than the opposite charge located at the same distance from the surface plane but inside the metal; this virtual charge is called the image charge or mirror charge, the Coulomb potential produced by the polarisation is called the image potential. For large distances outside the surface the image potential approaches the vacuum level. Many metals have a large energy gap at $\Gamma$ extending all the way up to the vacuum level. In this case a hydrogenlike series of bound states is expected within the potential $V(z)$ with eigen energies $E_{n}$ [116]:

$$
V(z)=\frac{\varepsilon_{r}-1}{\varepsilon_{r}+1} \frac{e^{2}}{4 \pi \varepsilon_{0} 4 z} \quad \text { and } \quad E_{n}-E_{\mathrm{vac}}=\frac{R_{\infty}}{16(n+a)^{2}}
$$


where $\varepsilon_{r}$ is the dielectric constant of the solid, $n$ the quantum number of the bound state, and $a$ the so-called quantum defect which takes the finite barrier height of the solid surface into account $[117,118]$. These so-called image-potentials states (IPS) are bound to the vacuum level $E_{\mathrm{vac}}$ and have a maximum binding energy of $1 / 16$ of the Rydberg constant $R_{\infty}$, i.e. $850 \mathrm{meV}$ for $n=1$ and $\varepsilon_{r} \rightarrow-\infty$. They were studied using momentum-resolved techniques like inverse photoemission [119] and $2 \mathrm{PPE}$ $[81,83,116,120,121]$.

Interestingly, a similar concept can be used to explain bound (and occupied) surface state on noble metal surfaces [118] as well as the interlayer states in $h-\mathrm{BN}$ : Using a $1 \mathrm{D}$ model potential along the normal of an isolated sheet of $h-\mathrm{BN}$, Blase et al. found that the $n=2$ IPS is fairly close to the first interlayer state in terms of charge distribution with respect to the atomic layer and binding energy with respect to the vacuum level [28]. This is shown in Fig. 9. It is anticipated that we find both, an interlayer state and an IPS for $h-\mathrm{BN} / \mathrm{Ni}(111)$ with similar dispersion and energies close to the energies given by Blase [92, 94]. Moreover, since both states can be brought into resonance their charge distribution must be very similar, what corroborates the physical picture of the interlayer states given in Ref. [28].

We will frequently refer to these states altogether as nearly-free-electron (NFE) states in the following.

\subsection{Unoccupied states in monolayer $h-B N$}

The unoccupied states in $h-\mathrm{BN} / \mathrm{Ni}(111)$ were studied using various methods. In one of the first publications from monolayer $h-\mathrm{BN}$, Nagashima et al. present secondaryelectron-emission spectra, which give access to conduction bands for energies above $E_{\mathrm{vac}}[53]$. The results are shown in Fig. 10 together with results of a previous DFT calculation [73]. The data show a region of high density of states starting at $\bar{\Gamma}$ at $5.5 \mathrm{eV}$ above $E_{\text {vac }}$, what corresponds to about $8.1 \mathrm{eV}$ with respect to $E_{F}$ (the workfunction of $h-\mathrm{BN} / \mathrm{Ni}(111)$ is $3.6 \mathrm{eV}$, see e.g. Ref. [54]). Van Hove singularities of $\sigma^{\star}$ and $\pi^{\star}$ states at $\bar{\Gamma}$ are likely to be at the origin of the high DOS observed in these spectra.

These findings are in line with results from near-edge x-ray absorption finestructure (NEXAFS) measurements [122, 123] and DFT calculations [65]. Briefly, in x-ray absorption, electrons from core levels are promoted into unoccupied states by absorption of a x-ray photon. The absorption strongly rises at the photon energy corresponding to the sum of the core level binding energy with respect to the Fermi energy $E_{F}$ and the energy of the unoccupied state above $E_{F}$. The energy of the unoccupied state is usually shifted due to attraction by the localised core hole. This shift therefore depends on the spatial extend of the final state wavefunction and often is difficult to estimate (see Ref. [124] for a detailed discussion of the core hole effects). Some experimental NEXAFS data are shown in Fig. 11: A sharp B1s ${ }^{-1} \pi^{\star}$ resonance was found at the B1s absorption edge, labelled $\mathrm{A}$ in the spectra. Recalling that the unoccupied $\pi^{\star}$ state at $\mathrm{K}$ is strongly localised at the boron site, the corresponding energy can be related to 


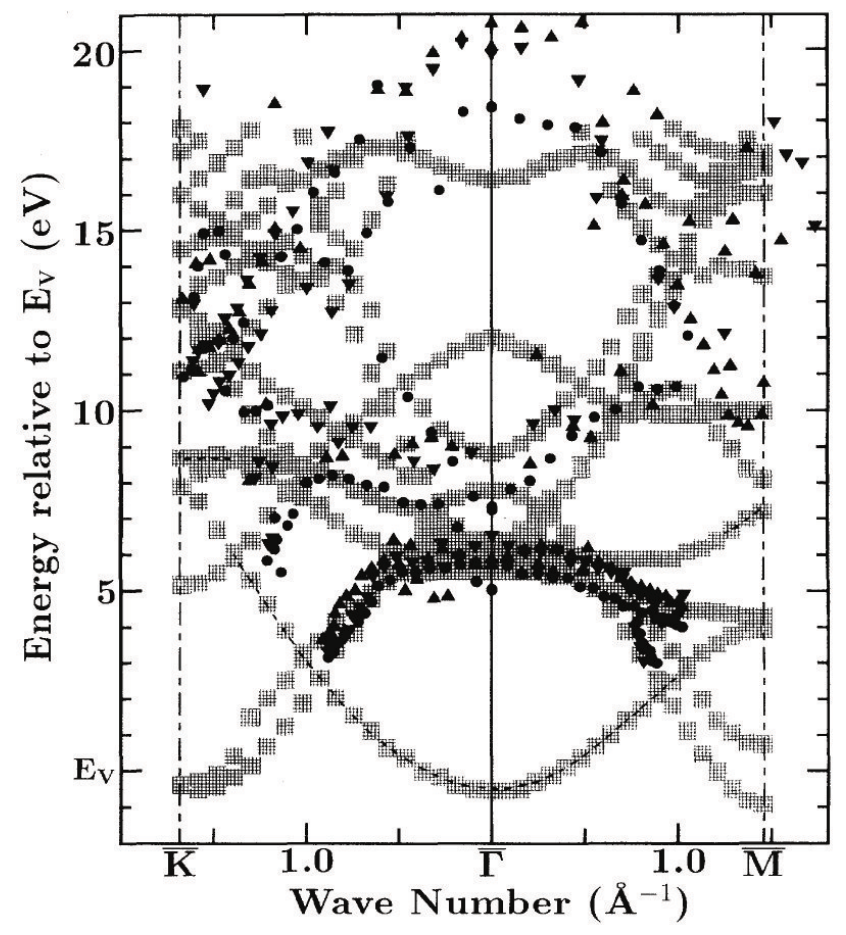

Figure 10. Dispersion of unoccupied states as obtained from secondary electron emission data for three surfaces and primary electrons with kinetic energies 60$110 \mathrm{eV}: h-\mathrm{BN} / \mathrm{Ni}(111)$ (circles), $h-\mathrm{BN} / \mathrm{Pd}(111)$ (upward triangles) and $h-\mathrm{BN} / \mathrm{Pt}(111)$ (downward triangles). The data are displayed against the energy with respect to $E_{\mathrm{vac}}$. The grey symbols are results from bandstructure calculations, and dashed lines indicate the lower NFE $\Gamma_{1}^{+}$state [73]. Reprinted with permission from Ref. [53]. (Copyright 1995 by the American Physical Society)

the $\pi^{\star}$ binding energy with respect to the Fermi level: Taking the change in Coulomb potential due to the core hole and screening into account the DOS and corresponding absorption spectra were calculated and compared to experiment: The partial p-derived DOS projected onto the boron site shows a strong maximum at about $2.0 \mathrm{eV}$ above $E_{F}$. Feature A is visible for all thicknesses from bulk to monolayer but with decreasing intensity and even vanishes in the monolayer limit for the B K-edge [122]. Its spectral weight is mostly transferred to the energy range below the onset of the $1 \mathrm{~s}^{-1}-\pi^{\star}$ resonance, i.e. for transitions from the respective 1s core state into states between the metal Fermi energy and the $\pi^{\star}$-band. This was interpreted as evidence for hybridisation between $\mathrm{Ni}$ d-states and $\mathrm{N} 2 \mathrm{p}$ states and indeed assigned to anti-bonding $\mathrm{Ni}-\mathrm{d}-\mathrm{Np} z$ hybrids as result of DFT calculations [65, 124]. Moreover, this assignment was corroborated by NEXAFS measurements taken in comparison from bulk $h-\mathrm{BN}, h-\mathrm{BN} / \mathrm{Cu}(111)$, and $h^{-}$ $\mathrm{BN} / \mathrm{Ni}(111)$ [125]: the $\mathrm{Cu}$ spectra strongly resemble spectra taken from bulk $h$-BN with only a very weak pre-edge feature indicating only small hybridisation with $\mathrm{Cu}$ states.

A second feature appears in the near-edge region, labelled $\mathrm{A}^{\prime \prime}$. Its weight relative to the $\pi^{\star}$-resonance increases from the bulk to the monolayer case and is slightly higher 

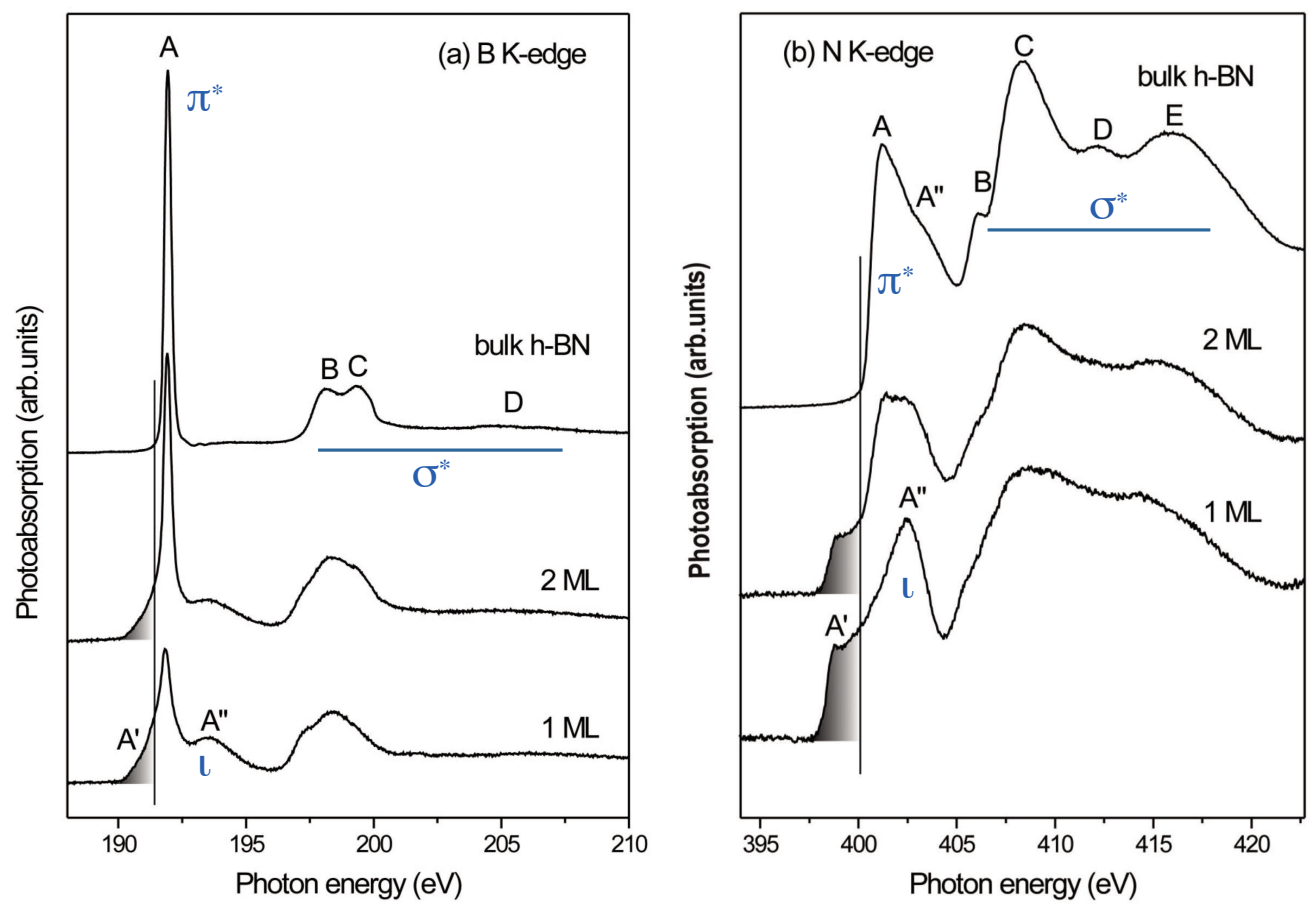

Figure 11. Near-edge x-ray absorption finestructure data from $h-\mathrm{BN}$ films on $\mathrm{Ni}(111)$ from a) B K-edge, b) N K-edge. Bulk $h$-BN, $2 \mathrm{ML}$, and $1 \mathrm{ML}$ correspond to thick bulk-like (> $20 \mathrm{~nm}), 2$ layers and one layer of $h-\mathrm{BN}$ deposited on Ni(111) by CVD. The letters $\mathrm{A}, \mathrm{A}^{\prime}, \ldots \mathrm{E}$ designate transitions which are explained in the text; the shaded area below the onset of the $\pi^{\star}$-resonance, labeled by $\mathrm{A}^{\prime \prime}$, denotes the pre-edge intensity. Blue labels are the assignments to the final states $\pi^{\star}, \sigma^{\star}$, and $\iota$ (interface state) of the involved transitions (see text for details). Reprinted with permission from Ref. [122]. (Copyright 2004 by the American Physical Society). The blue labels were added by us.

for the $\mathrm{N}$ than for the $\mathrm{B}$ resonance. It has roughly the same energy with respect to the resonance for the $\mathrm{B}$ and $\mathrm{N}$ K edges and is rather broad suggesting a delocalised state as origin [122]. It is attributed to transitions into interlayer (bulk) or interface (monolayer) states. The higher intensity for the N K-edge is in agreement with fairly strong contributions from N 2p states found by Catellani et al. [73]. Note again that its energy position cannot be directly compared to the energy of the $\pi^{\star}$-resonance due to different screening of the core hole produced.

The most direct way of measuring the dispersion again is given by angle-resolved inverse photoemission. Zumbrägel et al. measured the band structure above $E_{F}$ in $h-\mathrm{BN} / \mathrm{Ni}(111)$ and determined the dispersion of the NFE states at $\bar{\Gamma}$. Some selected spectra are displayed in Fig. 12(a) and the dispersion in (b). The lowest-energy state at $\bar{\Gamma}$ is found about $1.7 \mathrm{eV}$ above $E_{F}$ with electron-like dispersion and an effective mass of $m^{\star} \approx 1.1 m_{e}$ in units of the free-electron rest mass $m_{e}$ [92]. A second state with similar dispersion is observed with an energy of about $3 \mathrm{eV}$ with respect to the Fermi energy and an effective mass of $1.2 \mathrm{~m}_{e}$. The first state agrees in position and 

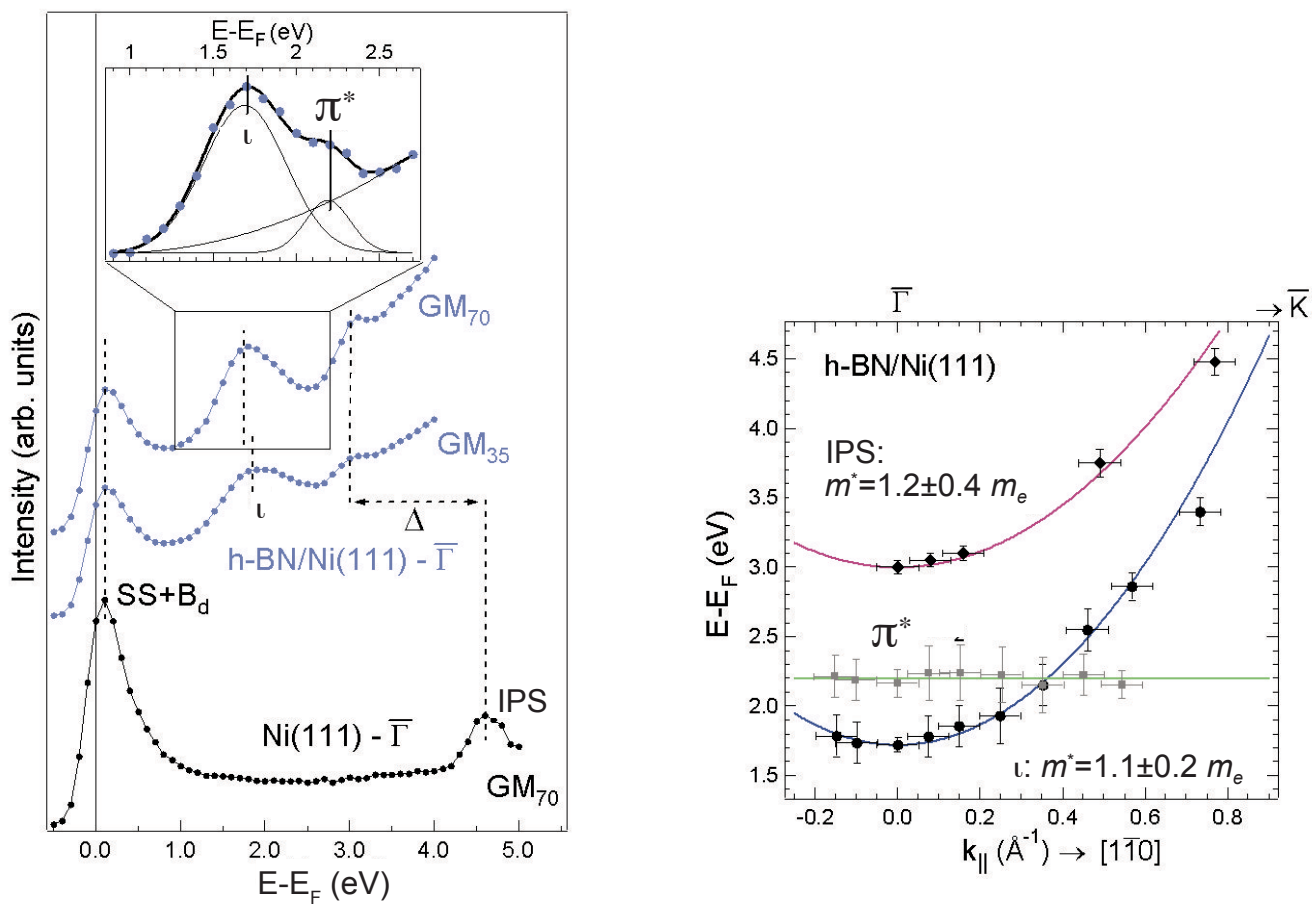

Figure 12. Inverse photoemission data from $h-\mathrm{BN} / \mathrm{Ni}(111)$. Left panel: normal incidence spectra for $h-\mathrm{BN} / \mathrm{Ni}(111)$ (blue symbols and lines) compared to $\mathrm{Ni}(111)$ (black symbols and line). The spectra were taken using two different Geiger counters $\mathrm{GM}_{35}$ and $\mathrm{GM}_{70}$. Inset: close-up of the interface peak taken with higher energy resolution. Right panel: dispersion of the three transitions IPS, $\pi^{\star}$, and $\iota$ as extracted from the angle-resolved data (symbols) and corresponding fits: the effective band masses resulting from parabolic fits are given in the graph. Reproduced and adapted from Ref. [92]. The peak labels were changed by us for the sake of consistency.

qualitative dispersion with the interface state $\iota$ predicted by Grad et al., but with a higher effective mass than in the calculation (theory: $m^{\star} \approx 0.73 m_{e}$ [102]). Within DFT, Huda and Kleinman compare the generalised gradient approximation (GGA) and local density approximation (LDA) to describe the adsorption and the bandstructure of $h-\mathrm{BN}$ on $\mathrm{Ni}(111)$ [113]. There are only little differences in the bandstructures resulting from both approximations; the occupied states are almost identical and can hardly be distinguished by comparison with ARPES data. The interface state, however, is found with both approximations, but at significantly higher energy within LDA (about $2.5 \mathrm{eV}$ above $E_{F}$ ) than within GGA, in which the interface states appears at about $1.7 \mathrm{eV}$ like in previous calculations and in the experiment.

Since there is only a single layer in monolayer $h-\mathrm{BN} / \mathrm{Ni}(111)$, we do not expect a second NFE interface state because the bulk $\Gamma_{3}^{-}$interlayer state appears at $\bar{\Gamma}$ due to back-folding of the lower state at the BZ boundary normal to the surface (point A in the 3D BZ, see Fig. 4). The bare Ni(111) surface supports an image potential state (IPS), which was found at about $750-850 \mathrm{meV}$ below $E_{\text {vac }}$, thus about $4.4-4.6 \mathrm{eV}$ above $E_{F}$ $[126,127]$. Taking into account the reduction in workfunction of $\Delta \phi \approx-1.8 \mathrm{eV}$ upon 
adsorption of the $h$-BN-layer [102], the second NFE state observed in the ARIPES data was assigned to an IPS in front of the $h-\mathrm{BN} / \mathrm{Ni}(111)$ surface [92]. The effective mass is close to the free-electron rest mass as expected and the binding energy is slightly reduced to about $650 \mathrm{meV}$ due to the presence of a dielectric layer which pushes the IPS further away from the metal surface. Laturia et al. calculated dielectric constants for different 2D materials and found that the response of single-layer $h$-BN to out-of-plane excitations is given by static and optical-frequency dielectric constants of $\epsilon_{\perp 0}=3.29$ and $\epsilon_{\perp \infty}=2.89$, respectively [128]. Based on these values, we would expect a reduction by a factor $(\epsilon-1) /(\epsilon+1) \approx 0.5$ [116], which yields a much smaller binding energy. The other way around, we can determine the dielectric constant which would lead to the observed binding energy of the IPS. This way we obtain $\epsilon=19$ which, interestingly, is in the order of the dielectric constant calculated for the bulk L-point of paramagnetic nickel [129]. Thus the simple picture of $h-\mathrm{BN}$ acting as dielectric layer is oversimplified, and, as we will see later in Section 7, the IPS still penetrates the Ni substrate to a certain extent. Giraud et al. calculated the bandstructure of $h-\mathrm{BN} / \mathrm{Ni}(111)$ using $13-$ layer slab calculations and actually found a second NFE state roughly at the correct position above the interface state [110]. The authors do not comment on the second state, and we conjecture that it might indeed be an IPS-like solution localised in the $15 \AA$ thick vacuum layer of the slab used by Griaud, sufficient to host a $n=1$ IPS, that extends typically $10 \AA$ into the vacuum [121].

Alongside the Ni-derived states in the ARIPE spectra close to $E_{F}$, which which are shown for $h-\mathrm{BN} / \mathrm{Ni}(111)$ and pristine $\mathrm{Ni}(111)$ in Fig. 12, a weak shoulder appears closely above the interface state. It is reminiscent of ARIPE spectra from single-crystalline graphite, in which all spectra show a weak non-dispersing feature at $1.8 \mathrm{eV}$ above $E_{F}$. It was assigned to a high DOS of the $\pi^{\star}$ band at M of bulk graphite [130]. In a similar way the non-dispersing peak at $2.2 \mathrm{eV}$ above $E_{F}$ observed in Ref. [92] was attributed to a van Hove singularity in the DOS at $\mathrm{K}$. This allows us to estimate the energy of the anti-bonding $\pi^{\star}$-band to be $2.2 \mathrm{eV}$ above the Fermi energy of nickel and, thereby, about $0.5 \mathrm{eV}$ above the conduction band minimum given by the interface state $\iota$. The energy difference is larger than predicted by DFT [102] but yields the correct energetic order for monolayer $h-\mathrm{BN}$.

\subsection{Quasiparticle bandstructure in monolayer $h-B N / N i(111)$}

From the previous results we may draw a combined bandstructure for electron removal (ARPES) and electron addition (ARIPES). Since the electron count changes in both spectroscopies by +1 or -1 , the final states in both spectroscopies are not eigenstates anymore and many-body effects may change the energy scales. We refer to this bandstructure as quasiparticle bandstructure. Care must be taken in comparing this bandstructure and the energy gaps to optical gaps and a joint density of states but they give the correct momenta of the band edges and the energy gap. The results for $h-\mathrm{BN} / \mathrm{Ni}(111)$ are shown in Fig. 13 together with bands calculated using ab initio DFT 


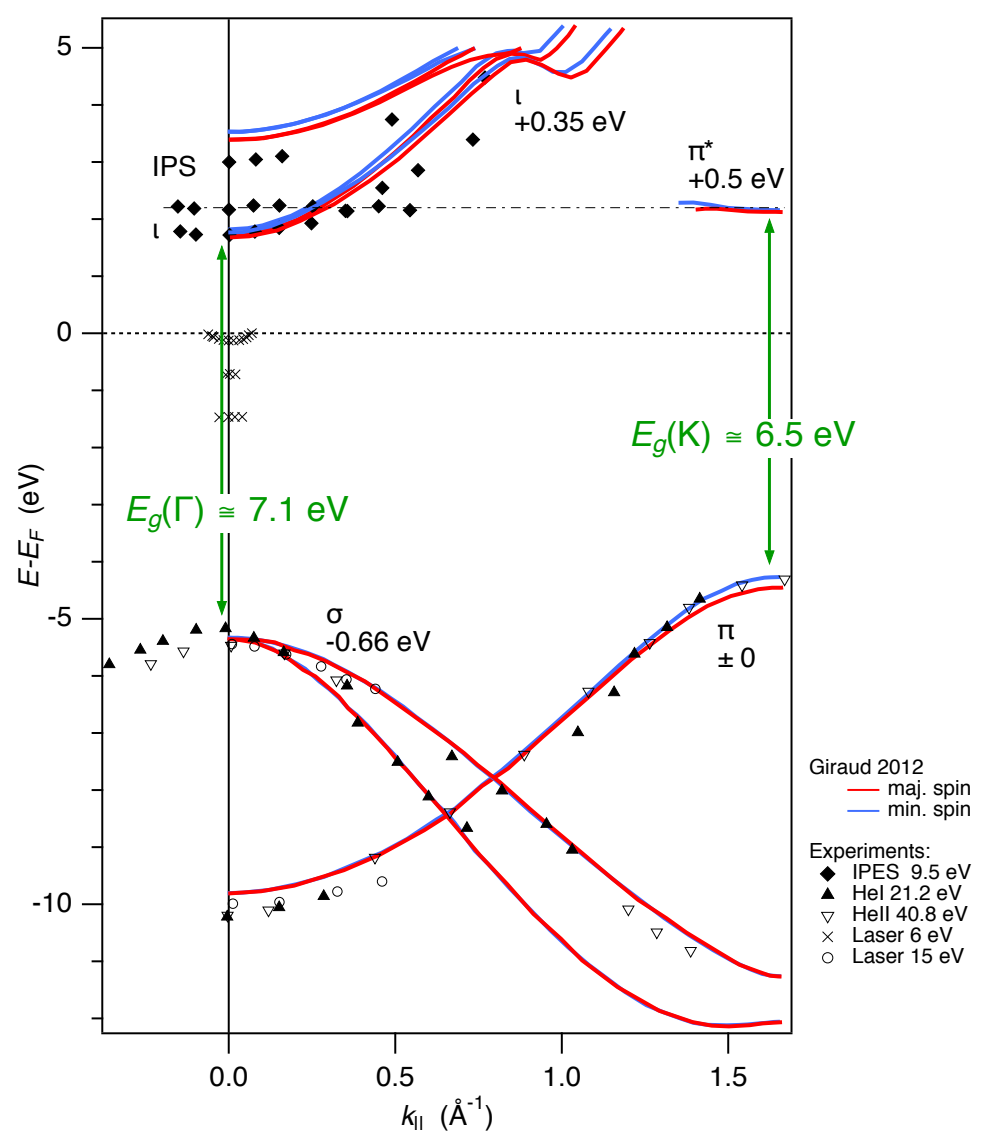

Figure 13. Compilation of results from angle-resolved (inverse) photoemission: Experimental data points (symbols) are plotted against the wavevector along $\overline{\Gamma K}$. The calculated energy bands for monolayer $h-\mathrm{BN}$ on top of a 13-layer Ni slab [110] were shifted and are shown as red and blue lines for majority and minority spin, respectively. The respected energy shifts are indicated as well as the two direct energy gaps at $\bar{\Gamma}$ and $\overline{\mathrm{K}}$. The data points are taken from the following references: ARIPES (solid diamonds) Ref. [92], ARPES (Laser and He-lamp) Ref. [90].

slab calculations [110]. The theoretical bands were shifted at high-symmetry points such as to comply with the experimental values. This way we obtain an estimate for the direct quasiparticle band gaps in $h-\mathrm{BN}$, which amount to $6.5 \mathrm{eV}$ and $7.1 \mathrm{eV}$ for $\overline{\mathrm{K}}$ and $\bar{\Gamma}$, respectively. This is smaller than the $\pi-\pi^{\star}$ direct gap of $7.25 \mathrm{eV}$ at $\overline{\mathrm{K}}$ resulting from recent DFT calculations including many-body $\mathrm{G}_{0} \mathrm{~W}_{0}$ corrections [109], whenever the overall agreement of the calculated single-layer bandstructure and our results is very good. 


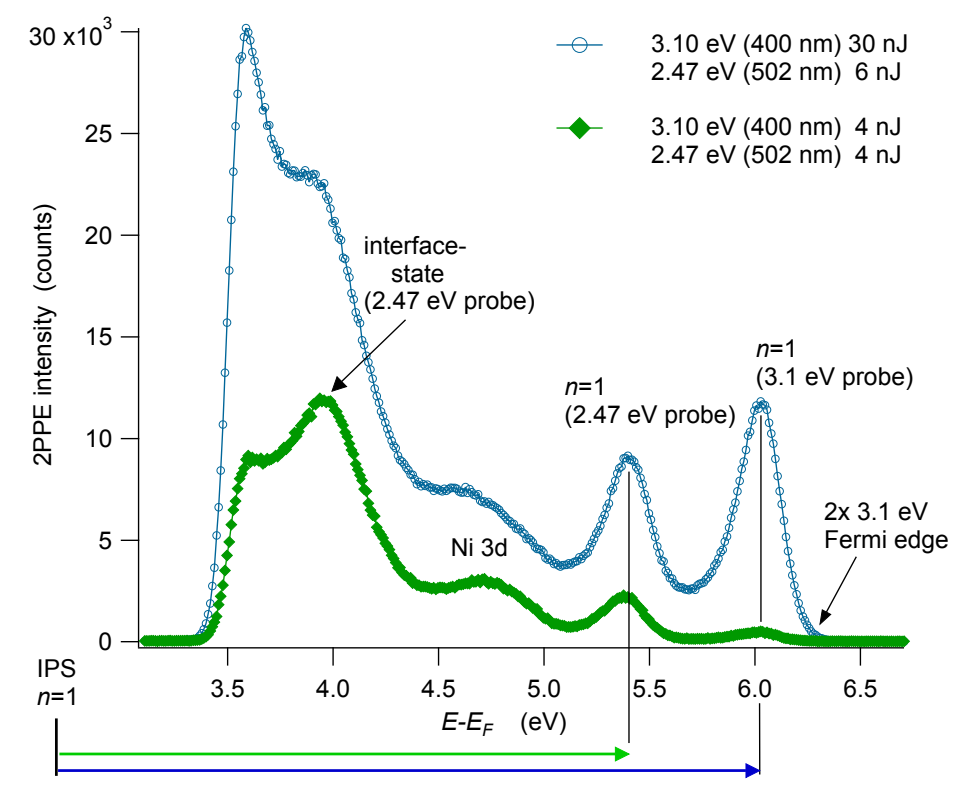

Figure 14. Two-colour 2PPE spectra from $h-\mathrm{BN} / \mathrm{Ni}(111)$, here $h \nu_{1}=3.1 \mathrm{eV}$ and $h \nu_{2}=2.47 \mathrm{eV}$ in temporal coincidence. The pulse energies are given, the two spectra differ by the ratio of the respective pulse energies $I_{1}$ and $I_{2}$ : blue open circles and line $I_{1} / I_{2}=6$, green solid diamonds and line $I_{1} / I_{2}=1$. The transitions are labelled; note that the IPS appears twice in each spectrum. Throughout this paper, we will use the final state energy scale with respect to $E_{F}$ for each $2 \mathrm{PPE}$ spectrum.

\section{Dynamics and lifetimes of electronic excitations}

\subsection{Two-photon-photoelectron (2PPE) spectroscopy}

Many data sets were taken from $h-\mathrm{BN} / \mathrm{Ni}(111)$ using different configurations for pump and probe beams like one- or two-colour experiments or different polarisations. A first and thorough study of intermediate state dispersion and excited state lifetimes was published by Muntwiler and co-workers in 2007 [94]. We will first focus on the peak assignments and resulting energies and effective masses. Note that we use the final state energy in 2PPE spectra in order to avoid any ambiguity. Intermediate state energies can be calculated from the spectra by subtracting the probe photon energy, initial state energies by subtracting both, pump and probe photon energies. Moreover, in order to identify the origin of or dominant contribution to the peak, the photon energy can be varied, as will be shown later in Fig. 15. The corresponding peak shift with varying pump and/or probe photon energy then allows the peak to be assigned to an initial, intermediate, or final state or even an Auger-like process [78]. Furthermore, the independent variation of pump and probe intensities lead to variations of the peak height in the spectra: in $2 \mathrm{PPE}$ the peak height is proportional to the product of pump and probe intensities and, thereby, together with the variation of its energy with photon energies helps in identifying the pump-probe sequence responsible for the peak in the 

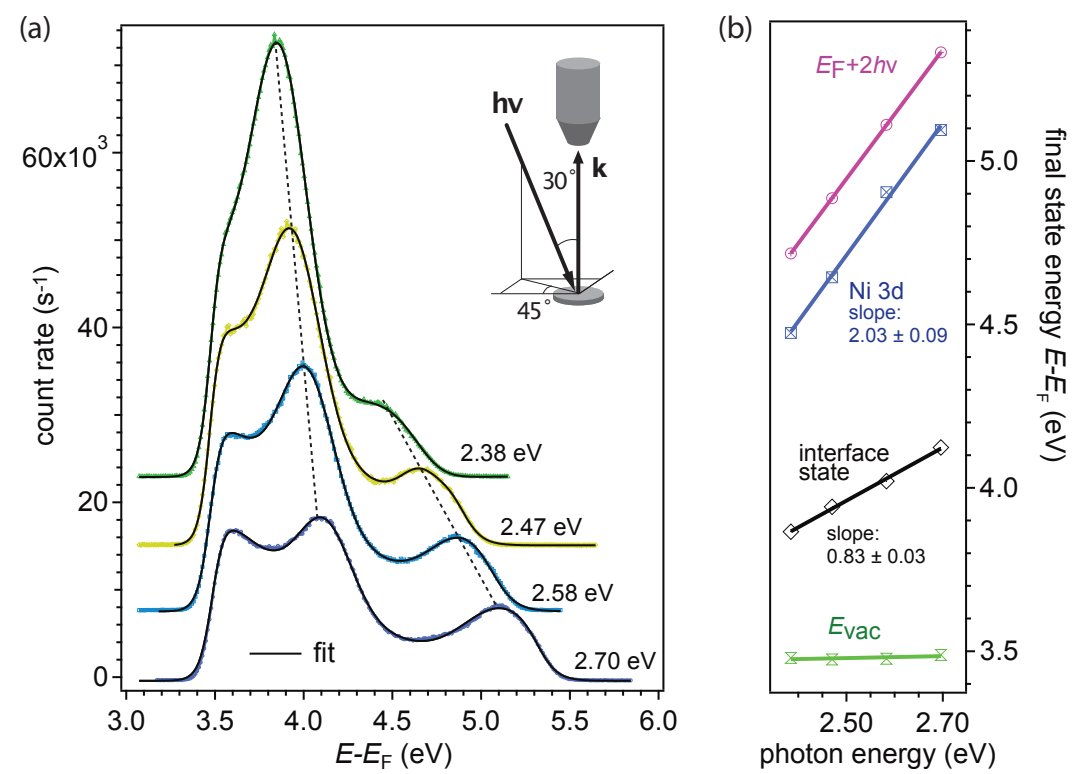

Figure 15. (a) Single-colour $2 \mathrm{PPE}$ spectra for photon energies between $2.70 \mathrm{eV}$ and $2.38 \mathrm{eV}$. The photon energy is too small to allow for population of the IPS, the two features are identified as direct $2 \mathrm{PPE}$ transition from the $\Lambda_{3}$ bulk d-bands of $\mathrm{Ni}$ and the interface state $\iota$. The spectra were normalized to the yield of the non-resonant Ni dband. (b) Final state energies corresponding to the secondary cut off (open triangles), the interface state (open diamonds), the $\mathrm{Ni} 3 d$-band (open squares) and the Fermi edge (open circles) as function of photon energy. Slopes of 1 or 2 in the linear fits (straight lines) indicate unoccupied or occupied states, respectively [88].

spectrum. Two previously unpublished example spectra taken later with the optical parametric amplifier (OPA) are shown in Fig. 14.

According to 2PPE experiments of Muntwiler et al. [94] and the inverse photoemission data shown in the preceding section [92], as well as photon-energy dependent data [88] we can assign the peaks in Fig. 14 to transitions out of Ni dstates (direct two-photon transitions), transitions via the interface state and via the IPS of $h-\mathrm{BN} / \mathrm{Ni}(111)$. The IPS appears twice due to the use of two different photon energies; the interface state can be recognized as strong peak when probed with the low photon energy close to resonance (see Fig. 15), but only as weak feature merged with the Ni d-peak when probed by $3.1 \mathrm{eV}$ light. Figure 15 shows single-colour spectra from the interface state for a few selected photon energies. The interface state transition moves with roughly once the photon energy and reaches its maximum intensity at $h \nu_{\text {res }}=2.38 \mathrm{eV}$. From the peak position and the photon energy the interface state energy can be calculated to be $1.50 \mathrm{eV}$, thus about $200 \mathrm{meV}$ less than results from inverse photoemission. One might think that interface state and IPS energies and, in particular, their effective masses depend strongly on sample quality and vary significantly between preparations. This is true to some extend but the state energies were found to scatter only by little over the years. A compilation of data for the two NFE states can be found 
in Table 1.

\begin{tabular}{|c|c|c|c|c|c|}
\hline \multirow[t]{2}{*}{ method } & \multicolumn{2}{|c|}{ interface state $\iota$ : } & \multicolumn{2}{|l|}{ IPS: } & \multirow[t]{2}{*}{ Ref. } \\
\hline & $\begin{array}{c}E_{\iota}-E_{F} \\
(\mathrm{eV})\end{array}$ & $\begin{array}{l}m_{\iota}^{\star} \\
\left(m_{e}\right)\end{array}$ & $\begin{array}{c}E_{\mathrm{IPS}}-E_{\mathrm{vac}} \\
(\mathrm{eV})\end{array}$ & $\begin{array}{l}m_{\mathrm{IPS}}^{\star} \\
\left(m_{e}\right)\end{array}$ & \\
\hline DFT & $1.75 \downarrow ; 1.63 \uparrow$ & $\approx 0.76$ & n.a. & n.a. & [102] \\
\hline IPE & $1.83 \downarrow ; 1.7 \uparrow$ & 1.1 & -0.65 & 1.20 & {$[92]$} \\
\hline 2PPE: & & & & & \\
\hline $2 \mathrm{x} 2.95 \ldots 3.15 \mathrm{eV}$ & 1.46 & & -0.77 & & 2001 (unpubl.) \\
\hline $2 \mathrm{x} 3.1 \mathrm{eV}$ & 1.51 & 0.43 & -0.65 & 1.12 & {$[94]$} \\
\hline $1.46+2.92 \mathrm{eV}$ & 1.51 & & -0.65 & & {$[94]$} \\
\hline $2 \mathrm{x} 2.38 \ldots 2.7 \mathrm{eV}$ & 1.50 & 1.52 & & & [88], this work \\
\hline $2.47+3.1 \mathrm{eV}$ & 1.50 & & -0.57 & & this work \\
\hline $1.49+5.99 \mathrm{eV}$ & 1.48 & & & & {$[90]$} \\
\hline
\end{tabular}

Table 1. Compilation of energies and effective masses of the NFE-like interface state and the image-potential state (IPS) at $\bar{\Gamma}$, as measured by 2PPE in different configurations, and compared to inverse photoemission and DFT calculations. The arrows $\downarrow$ and $\uparrow$ indicate the two spin orientations, $\uparrow$ meaning majority spin. Note the different reference energies for $\iota$ and IPS.

These results give a fairly consistent picture together with inverse photoemission data: around $\bar{\Gamma}$, two NFE-like states are found, the one at lower energy being assigned to the interface state, the one at higher energy to an image-potential state. Two points, however, are striking:

(i) The energy of the interface state is systematically found to be lower by about $200 \mathrm{meV}$ in $2 \mathrm{PPE}$ experiments than in calculations $[102,110]$ and inverse photoemission [92].

(ii) The very small value of the effective mass $m_{\iota}^{\star}=0.43 m_{e}$ of the interface state as found in two-colour experiments by Muntwiler et al. [94].

Concerning the second point, one might argue that the exceptionally small effective mass is due to two states which contribute with momentum dependent intensity and thus mimic a single steeply dispersing band. Since the exchange-split pair of interface states was never resolved in 2PPE so far, and both bands are supposed to have parallel dispersion, this picture seems to be rather improbable. In Fig. 16 we plotted data from inverse photoemission, $2 \mathrm{PPE}$ in resonance and from the original work of Muntwiler et al., and results of DFT together. The data of Ref. [94] fall on top of other 2PPE data close to normal emission $k_{\|}=0$, which are about $200 \mathrm{meV}$ below the actual interface state dispersion from IPES and DFT. Away from normal emission, the data are close to the dispersion as found in IPES and DFT. Taken together the dispersion strongly 


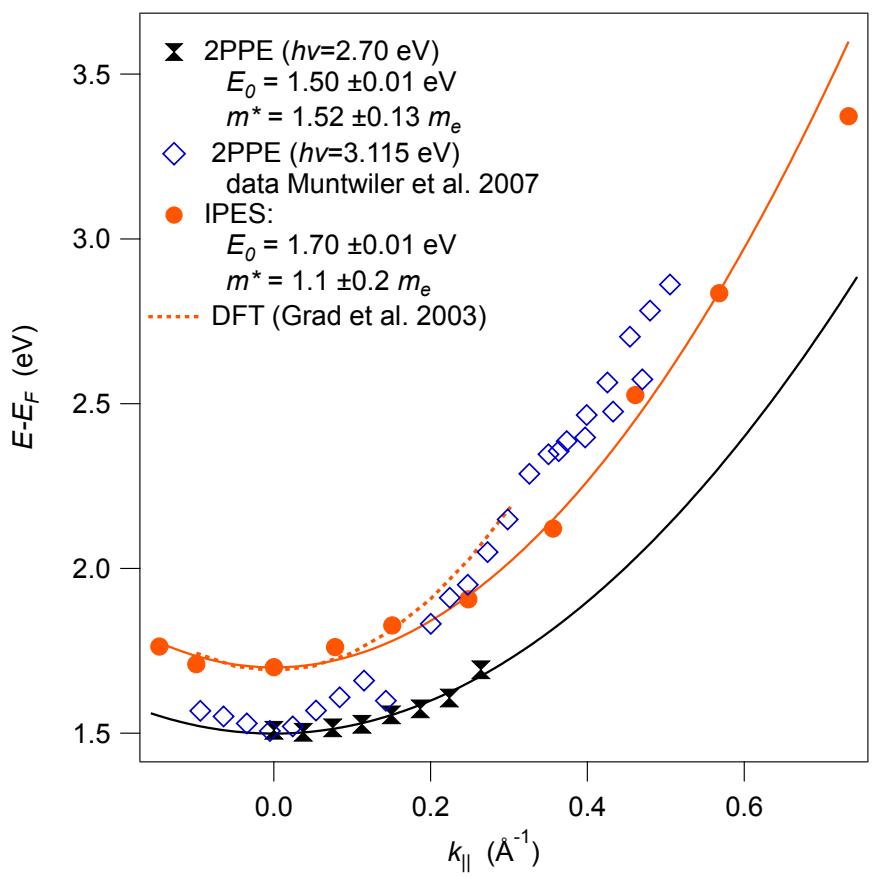

Figure 16. Single-colour $2 \mathrm{PPE}(h \nu=2.7 \mathrm{eV}$, black solid symbols) [88]) of $\iota$ as function of parallel momentum, together with 2PPE data from Ref. [94] (blue open diamonds), inverse photoemission data (solid orange circles [92]), and the DFT dispersion (dashed orange line, spin-averaged [102]). The solid lines are parabolic fits, the fit results are given in the legend. Note that the data of Muntwiler et al. were taken in a different azimuth along $\overline{\Gamma M}[94]$.

deviates from a single parabola as already noted by the authors of Ref. [94] and could emulate a much lighter mass than the actual band mass.

The main difference between 2PPE and IPES is that the first step of the two-photon process generates a polarisation of the system and a population of an unoccupied state without altering the total number of electrons, while IPES always adds an electron to the system and probes the energy of this additional electron as is sketched in Fig. 17. If stable excitons can be formed by absorption of a photon in the first step of $2 \mathrm{PPE}$, the energy of the electron promoted into the conduction band will be smaller than the actual conduction band energy. In the second step of $2 \mathrm{PPE}$, this level is then ionised and the exciton binding energy is taken from the photoelectron. Thus, if there are excitons, they can be seen by 2PPE [131, 132] but not by IPES, and the energy difference in 2PPE and IPES should give the exciton binding energy. Prada et al. calculated exciton binding energies for 2D materials, taking phosphorene as example [133]. The exciton binding energy $E_{X}$ depends on the inverse dielectric constant and the spatial extent, i.e. the average distance between hole and electron. In our model, the photohole is in the surface layer of $\mathrm{Ni}$ and the electron is localised in the interface state inbetween the $\mathrm{Ni}$ surface layer and $h-\mathrm{BN}$. Thus, the dimension of the exciton would be of the order of $a_{X} \leq 1 \mathrm{~nm}$. The use of a simple hydrogen-like Coulomb potential is strictly valid 


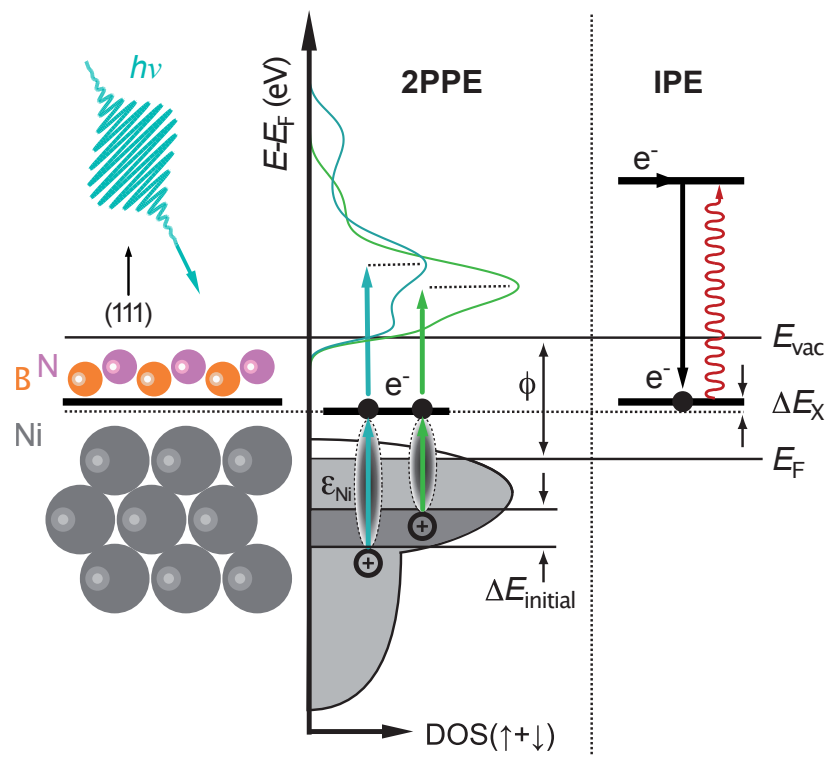

Figure 17. Sketch of the exciton model for the interface state at $\bar{\Gamma}$. From left to right: real space model of the system, DOS picture of $h-\mathrm{BN} / \mathrm{Ni}(111)$ with exciton formation by absorption of the first photon in 2PPE, and schematic view of the IPES process which adds an electron to the system. Taken from Ref. [88].

only for large excitons but might be suitable here to get a first estimate of the order of magnitudes: If we use $E_{X}=0.2 \mathrm{eV}$ and $\epsilon_{\perp} \approx 19$ from the binding energy of the IPS, we obtain [133]:

$$
\begin{aligned}
& a_{X}=-\frac{e^{2}}{4 \pi \epsilon_{0} \epsilon_{\perp} E_{X}} \approx 2 \AA \quad \text { and } \\
& \mu_{X}^{\star} / m_{e}=\frac{a_{0} \epsilon_{\perp}}{2 a_{X}} \approx 2.6,
\end{aligned}
$$

where $\epsilon_{0}, a_{0}$, and $\mu_{X}^{\star}$ denote the vacuum permittivity, the Bohr radius, and the reduced mass of the exciton, respectively. The size seems to be too small and the effective mass too large by about $70 \%$ with respect to the $2 \mathrm{PPE}$ results in Table 1, but they give already the correct order of magnitude. Moreover, the exciton would be stable for small center-of-mass momenta until its energy reaches the energy of the band edge. It could thus only be observed close to $\bar{\Gamma}$. The resulting dispersion corresponds quite well to the dispersion observed by Muntwiler et al. which is plotted in Fig. 16.

Finally the measurement of resonance energies for excitations into $\iota(\approx 2.38 \mathrm{eV}$, Fig. 15) and IPS ( $\approx 3.07 \mathrm{eV}$, data not shown) allows the initial state energies to be determined, as well. For the time being we only mention that the low photon energies used in our experiments preclude any contribution from $\sigma$ or even $\pi$ bands at $\bar{\Gamma}$. The initial states are found in the quasi-continuum of the $\mathrm{Ni}$ d-bands, and we will make use of this fact later on in Section 7. For being able to resonantly excite electrons from $\mathrm{Ni}$ d- into the interface or IPS, the latter must have significant wavefunction overlap 

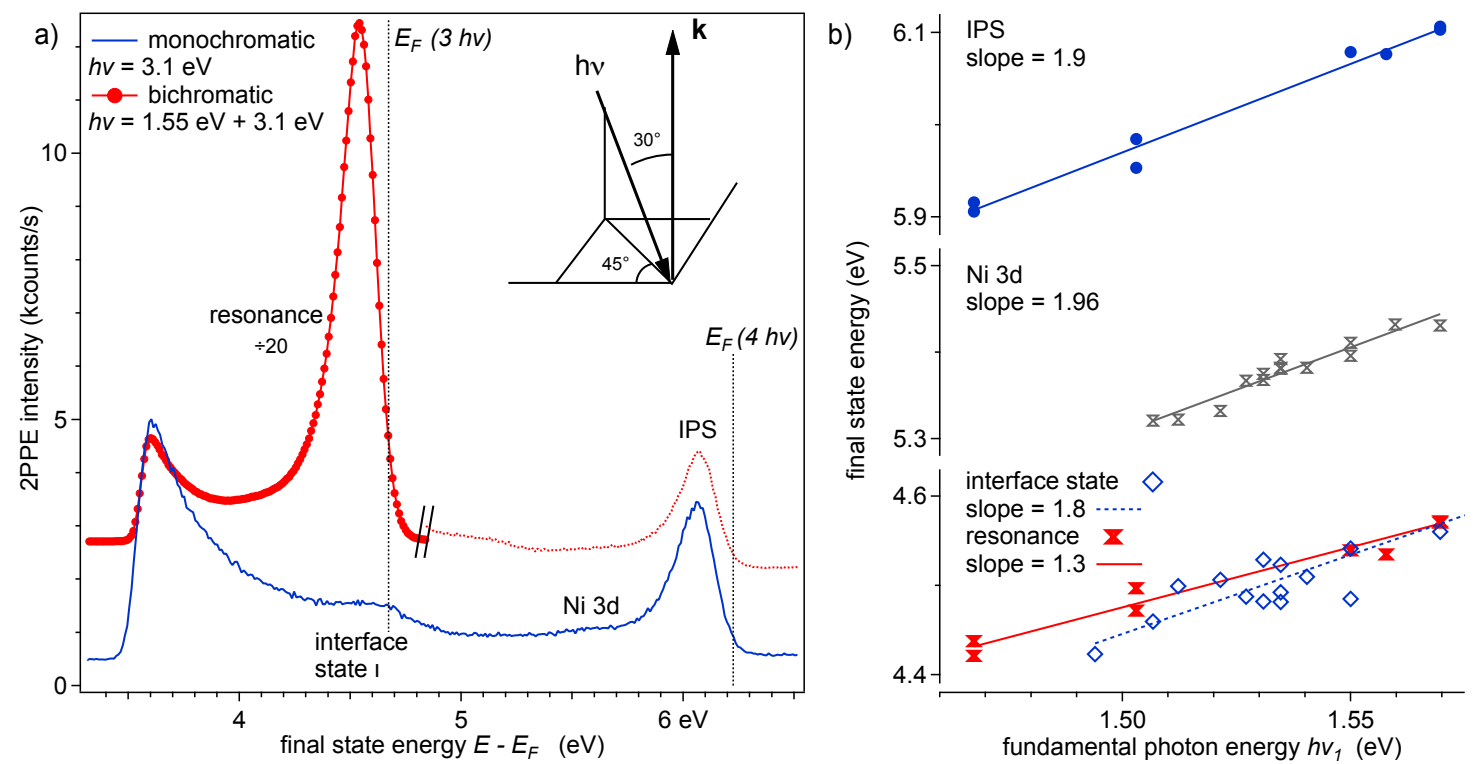

Figure 18. Two-colour $2 \mathrm{PPE}$ spectra from $h-\mathrm{BN} / \mathrm{Ni}(111)$. a) The upper (red) curve is taken with both light pulses, the fundamental $\left(h \nu_{1} \approx 1.55 \mathrm{eV}\right)$ and second harmonic $\left(h \nu_{2} \approx 3.1 \mathrm{eV}\right)$ in coincidence, i.e. at zero time delay. The lower (blue) curve shows the spectrum taken with only the second harmonic. Note that the low-energy part of the bichromatic spectrum was downscaled by a factor of 20 . Inset: the measurement geometry with beam incidence and electron detection direction $\mathbf{k}$. b) Dependence of four peak positions on the photon energy with line fits. Adapted from Ref. [94].

with the d-states and therefore penetrate into the substrate to a certain point, which corroborates the exciton picture for $\iota$ explained above and the high screening constant $\epsilon_{\perp}$ found for the IPS.

We emphasise that the conclusions drawn from our model cannot be directly applied to the case of bulk $h-\mathrm{BN}$. The optical properties of bulk $h-\mathrm{BN}$ are believed to be determined by excitons in the $\pi^{\star}$-bands at the border of the BZ $[33,37]$. Galvani et al. recently studied excitons in $\pi^{\star}$-states of single-layer $h-\mathrm{BN}$ by tight-binding calculations [109]: The ground state exciton at $\overline{\mathrm{K}}$ was found to be strongly localised with a huge binding energy of about $2 \mathrm{eV}$. In contrast to our experiments, however, only $h-\mathrm{BN}$ contributes to the screening $(\epsilon \approx 3[109])$. In our case, the photohole is efficiently screened by the metal, what reduces the binding energy by almost one order of magnitude in agreement with the results of our simple model above.

\subsection{Lifetime measurements by time-resolved 2PPE}

In order to get a complete picture about carrier dynamics in this $2 \mathrm{D}$ material, it is advantageous to use real-time techniques. Besides the temporal information 2PPE offers momentum resolution and is, thereby, complementary to time-resolved optical probes like transient absorption spectroscopy, which is often used in the context of electrontransfer processes across interfaces. Muntwiler and co-workers [94] used the fundamental 


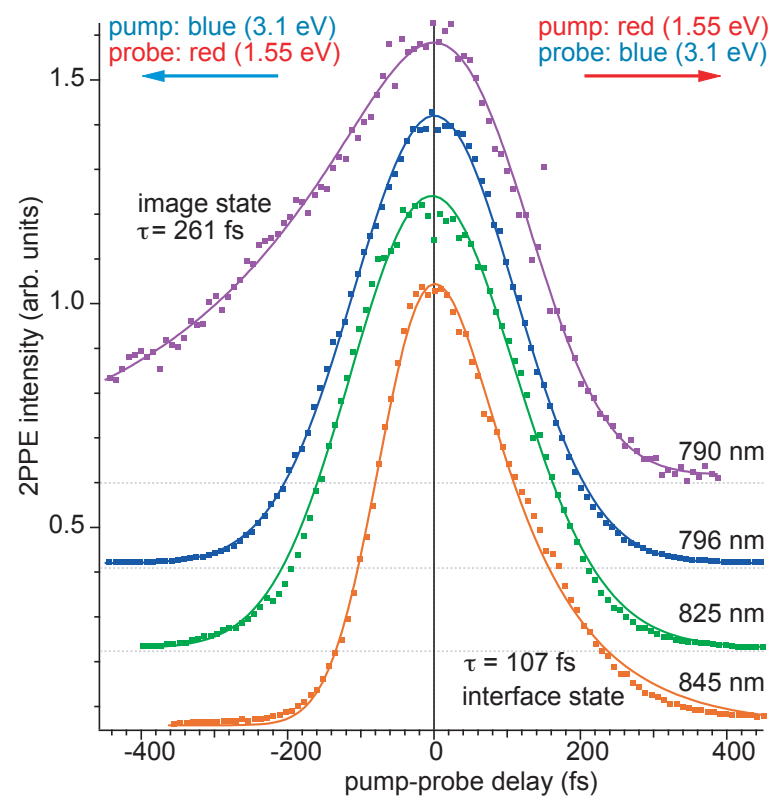

Figure 19. 2PPE Transients on resonance for four different excitation wavelengths. The fundamental and the second harmonic are used for $2 \mathrm{PPE}$ and the fundamental wavelengths are indicated. For both directions of the delay axis, the sequence of photons is displayed at the top, the pump always preceding the probe. The spectra were offset vertically for the sake of clarity. The two traces at $790 \mathrm{~nm}$ and $845 \mathrm{~nm}$ could be fitted using rate equations and the corresponding time constants $\tau$ are given. Adapted from Ref. [94].

of the laser (infrared, IR of $1.55 \mathrm{eV}$ ) as pump and the second harmonic (blue, $3.1 \mathrm{eV}$ ) as probe for $2 \mathrm{PPE}$ measurements from $h-\mathrm{BN} / \mathrm{Ni}(111)$. Due to the workfunction of $3.6 \mathrm{eV}$ three infrared photons are necessary to produce a photoelectron. At the low fluences used in these experiments, these processes could be neglected and the spectra far from time delay zero were identical to the single-colour 2PPE spectra with blue light. In coincidence at delay zero, however, the spectra changed dramatically as shown in Fig. 18. While the IPS and the direct two-photon transition from Ni-3d do not depend on delay, a huge resonant peak is observed in coincidence at roughly the same position as the interface state for large delays [94].

The transients of the resonance intensity are plotted in Fig. 19 for four different wavelengths across the resonance. Above and below the resonance the maximum intensity rapidly drops and the pulse-pulse cross-correlation function becomes asymmetric. The key is the direction of the asymmetry as any real intermediate state causes an exponential decay for positive delays with respect to the corresponding pump pulse. Thus, the fact that the asymmetry changes sign means that we deal with two competing processes, once with the pulse sequence infrared pump - blue probe, once with the opposite sequence blue pump - infrared probe. The authors attribute the former processes to $2 \mathrm{PPE}$ via the interface state, the latter via the IPS. The resonance condition is fulfilled if the fundamental photon energy matches the energy difference of 

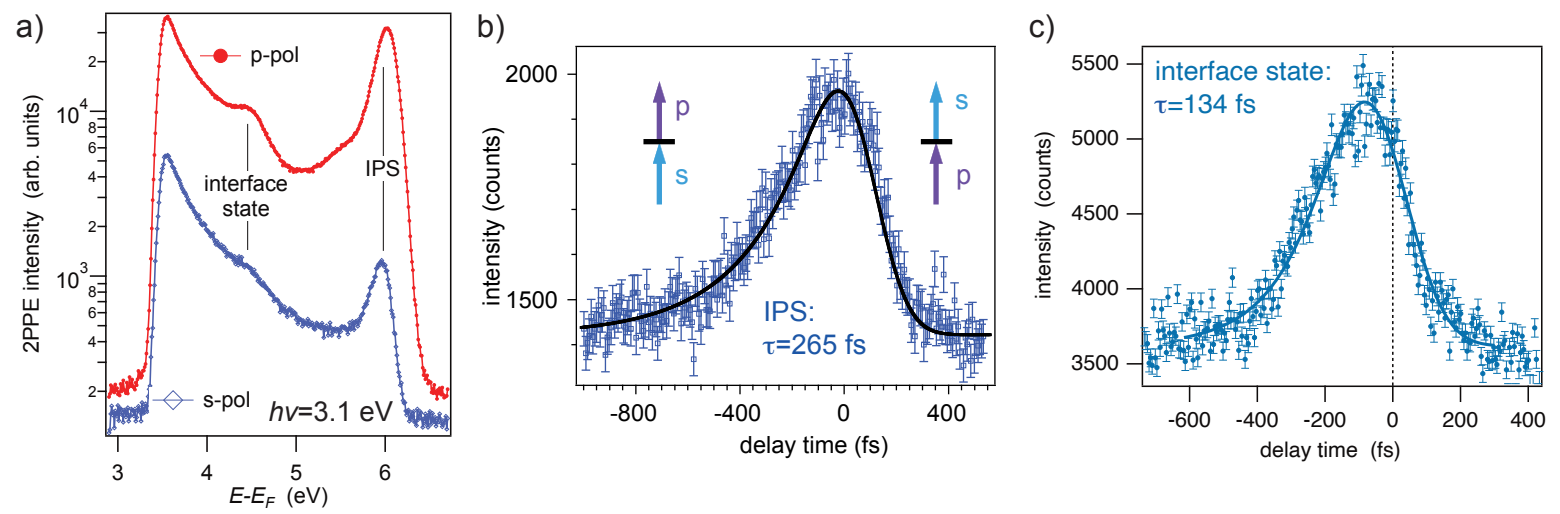

Figure 20. (a) Time-resolved single-colour 2PPE spectra taken at $h \nu=3.1 \mathrm{eV}$ with sand p-polarised light pulses [88, 95]. The time sequence is indicated in b) for negative and positive delays. a) Spectra for s- (blue open symbols) and p-polarised (red solid circles) light on a logarithmic intensity scale. The states can be populated using both polarisations but probed only with p-polarised light. b) and c) Transients for IPS and $\iota$, respectively. Lines show fits of rate equations, the resulting time constants are given.

interface and image-potential state (about $1.5 \mathrm{eV}$ ). In resonance the cross-correlation function is rather broad, and lifetimes can only be extracted out of resonance: according to this model, the authors find surprisingly long lifetimes of 107 fs and 261 fs for the interface state and the IPS, respectively [94]. In resonance, two channels for two-photon processes are available leading from the same initial state to the same final state and, thus, to resonant enhancement of the $2 \mathrm{PPE}$ peak intensity.

In order to corroborate this model, Leuenberger et al. used a blue-only two-photon sequence of pulses of orthogonal polarisation. As shown in Fig. 20a, both states appear much stronger when excited with p-polarised light due to dipole selection rules in the probe process [79]. Both states, however, can be populated with s-polarised light as well. The pulse sequence thus is asymmetric and the population decay should be observed for s-polarised pump and p-polarised probe, as can indeed be seen in Fig. 20b and c. Fitting the transients with rate equations yields $134 \mathrm{fs}$ and $265 \mathrm{fs}$, slightly longer but in excellent agreement with the findings of Muntwiler et al. [88, 95]. The lifetimes seem to be surprisingly long, in particular for the interface state which is close to the surface of the open d-shell metal nickel. Electron-electron scattering should be strong in this case leading to short lifetimes as observed from IPS on bare nickel [134]. We think that the electron trapped in the interface state is stabilised by interaction with the photohole leading to an exciton with a binding energy of $200 \mathrm{meV}$ and a lifetime a more than 100 fs.

Finally we wish to add that the spectra including the resonance process can be modelled in numerical simulations using a set of nine coupled differential equations within the density matrix formalism of the optical Bloch equations [85, 86]. A detailed description is beyond the scope of this work. As a result, the simulations yield dephasing times of the order of 6-8 fs in good agreement with dephasing times measured on bare 
$\mathrm{Ni}(111)[134]$ and thus complete our picture of the resonance process.

\section{Magnetic properties of and magnetisation dynamics in $h-\mathrm{BN} / \mathrm{Ni}(111)$}

The sensitivity of the NFE states in $h-\mathrm{BN}$ monolayers to the dielectric properties of the metal substrate was already demonstrated in the last sections. The goal of this section is to show that these states are even sensitive to the magnetic state of the underlying metal lattice.

Nickel is the showcase of itinerant band ferromagnetism, which was first described by Stoner [135, 136]: the simultaneous existence of strong Coulomb interaction in the d-shell and a high density of states at $E_{F}$ due to empty d-states leads to the appearance of spontaneous long-range magnetic order below a critical temperature, the Curie temperature $T_{c}$. In nickel, the spin degeneracy is lifted and the bands with minority spin polarisation, referred to sometimes as "spin down", are shifted to higher energy. The energy difference between bands of majority and minority spin is called the exchange splitting. As a result the d-bands of majority character are filled and all below $E_{F}$, while the minority band cross the Fermi energy. The holes in the minority bands result in a non-vanishing macroscopic magnetisation of the solid. The sp-bands are split as well but their contribution to the ferromagnetic order is negligible; hence they usually are considered as spectator states. It was shown amongst others by ARPES that the exchange splitting of the d-bands and sp-bands vanishes when the temperature approaches the Curie point [137, 138]; it can thus be used as an order parameter for ferromagnetism.

Due to the lifted spin degeneracy, the band edges of the gaps in the majority and minority bandstructures are at different energies. As a consequence, the reflection phase and, thereby, the binding energy of electrons in image-potential states will depend on their spin state [117]. Indeed, in 1992 Passek and Donath reported the observation of induced exchange splitting of $18 \mathrm{meV}$ for an IPS at room temperature in spin-polarised inverse photoemission data [127]. 2PPE experiments with high energy resolution and without spin detection were carried our but could not resolve the exchange splitting in nickel [126], but on different materials like iron by polarisation dependent 2PPE [139]. In spin-resolved 2PPE studies [140, 141] different lifetimes for majority and minority IPS on ferromagnetic Fe and Co thin films were observed, which gave an insight into quasi-elastic and inelastic scattering processes. From this it can be concluded that states bound in the interlayer or surface region are sensitive to and therefore suitable probes of the near-surface magnetisation.

Grad et al. computed the spin-dependent bandstructure of $h-\mathrm{BN} / \mathrm{Ni}(111)$ by means of slab calculations using DFT [102]: Besides the exchange-split bandstructure of nickel, the bands of $h-\mathrm{BN}$ were found to depend on the spin. More precisely, the $\pi$ band shows a sizeable exchange splitting at $\overline{\mathrm{K}}$, because the states there are dominated by $\mathrm{N}-\mathrm{p}_{z}$ states which hybridise with $\mathrm{Ni}$ d-orbitals. On the contrary, the in-plane $\sigma$-states show only very small splittings of the order of $10 \mathrm{meV}$. Since, as a consequence of $h-\mathrm{BN}$ 


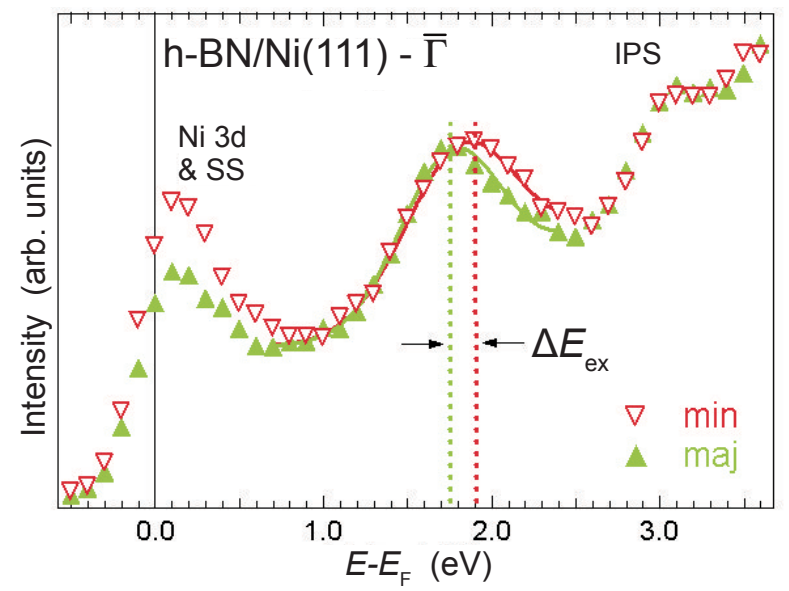

Figure 21. Spin-polarised inverse photoemission from $h-\mathrm{BN} / \mathrm{Ni}(111)$ at $300 \mathrm{~K}$, green upward triangles for majority spin, red downward triangles for minority spin polarisation. The exchange splitting $\Delta E_{\text {ex }}$ of $\iota$ is indicated by vertical dashed lines. Reproduced from Ref. [92]. The peak labels were added by us.

adsorption, charge is donated from $\mathrm{N}-\mathrm{p}_{z}$ into empty $\mathrm{Ni}$ d-states, the magnetic moment per $\mathrm{Ni}$ atom is slightly reduced from about $0.65 \mu_{\mathrm{B}}$ in bulk Ni to $0.6 \mu_{\mathrm{B}}$ at the surface. The bare $h$-BN-derived states are either entirely filled or empty and do not contribute to the magnetisation. Hence, $h-\mathrm{BN}$ cannot be considered magnetic.

For the interface state, an induced exchange splitting of $120 \mathrm{meV}$ was predicted with majority spin states having lower energy like in bulk nickel [102]. This splitting was confirmed by spin-polarised inverse photoemission [92]. In such experiments, a beam of partially spin-polarised electron is impinging on the surface and decays into low-energy states under radiation of photons [93]. From the calibrated spin polarisation of the beam and the photon spectra, spin-resolved spectra can be extracted as shown in Fig. 21. A splitting of $130 \mathrm{meV}$ was found for the interface state in excellent agreement with the theoretical predictions [92].

In view of probing magnetisation dynamics by monitoring the NFE states, one has to consider that the intense pump light in such experiments may lead to strong polarisation effects within the NFE states and that concomitant energy or intensity changes mask the signal of interest as seen from the resonance effect in the preceding section [94]. Hence, we chose the single-colour 2PPE transition via the IPS using $3.14 \mathrm{eV}$ light as probe, as sketched in Fig. 22. The position of the corresponding IPS peak was measured by means of $2 \mathrm{PPE}$ for different temperatures up to and through the Curie temperature of bulk $\mathrm{Ni}\left(T_{c}=631 \mathrm{~K}\right)$. This was done for two different samples, a bulk single crystal and a $80 \mathrm{~nm}$ single-crystalline film grown on sapphire.

We note in passing that the $2 \mathrm{PPE}$ spectra were found to differ slightly between the film and crystal samples. The workfunction of the film $(3.855 \mathrm{eV})$ is higher than the one of the crystal sample $(3.6 \mathrm{eV})$. This is likely due to the high defect density in the film which compromises the quality of the $h-\mathrm{BN}$ monolayer. Moreover, the secondary 

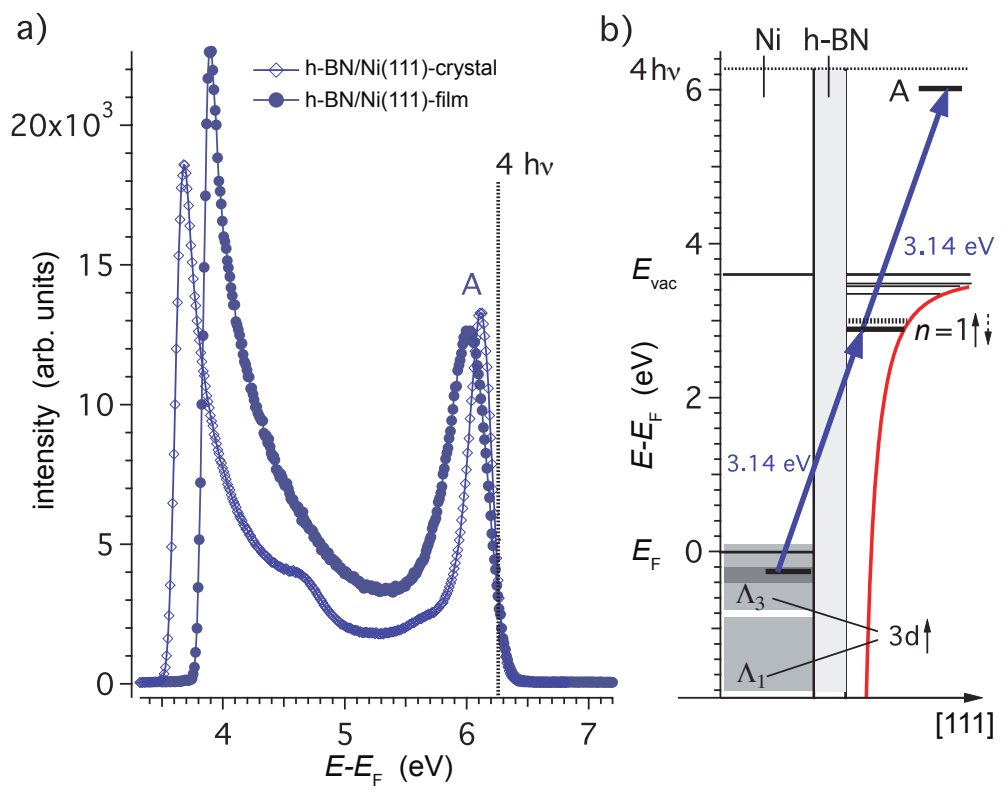

Figure 22. Study of induced exchange splitting of the IPS on $h-\mathrm{BN} / \mathrm{Ni}(111)$. a) Monochromatic $(h \nu=3.14 \mathrm{eV}) 2 \mathrm{PPE}$ normal emission spectra from monolayer $h-\mathrm{BN}$ on a $\mathrm{Ni}(111)$ bulk crystal (open diamonds) and on a $80 \mathrm{~nm}$ thick $\mathrm{Ni}(111)$-film on a $\mathrm{Al}_{2} \mathrm{O}_{3}(0001)$ substrate. b) Schematic sketch of the observed 2PPE transition from the majority Ni-3d band through the $n=1$ majority IPS as intermediate state. From Ref. [88].

electron peak at the low-energy cut-off is enhanced for the film sample, masking entirely the small interface state peak at $4.7 \mathrm{eV}$ above $E_{F}$. Yet, the IPS is well visible for both samples and seems to be robust against defects present in the thin film sample.

The peak position of the IPS is plotted in Fig. 23 against the sample temperature. For both types of samples a kink close to $T_{c}$ is observed, which clearly indicates the magnetic phase transition at the Curie temperature. Above $T_{c}$ the peak further shifts linearly with temperature towards higher energies. The slopes of $0.122 \mathrm{meV} / \mathrm{K}$ for the single crystal and $0.057 \mathrm{meV} / \mathrm{K}$ for the nickel film agree well with a thermally induced energy shift, as previously observed by photoemission from the Shockley surface state on $\operatorname{Ag}(111)$ [142]. These Shockley states can be modelled qualitatively like $n=0$ image potential states and their response to changes in the metal bandstructure should therefore be very similar [118]. The difference in slopes between the crystal and the film can at least partially be rationalised by invoking the differences in thermal lattice expansion of nickel and the sapphire substrate of the film.

Below $T_{c}$, the changing exchange splitting contributes to the change in peak position, thus producing a kink in the curve. Since the peak position in the spectra is dominated by the energy position of the intermediate state, we may deduce a pure magnetic shift of the IPS by subtracting the extrapolated thermal shift from the total one. The result is then fitted to the magnetisation curve of nickel [143]:

$$
M(T) / M(0) \propto\left(T_{c}-T\right)^{\beta}
$$



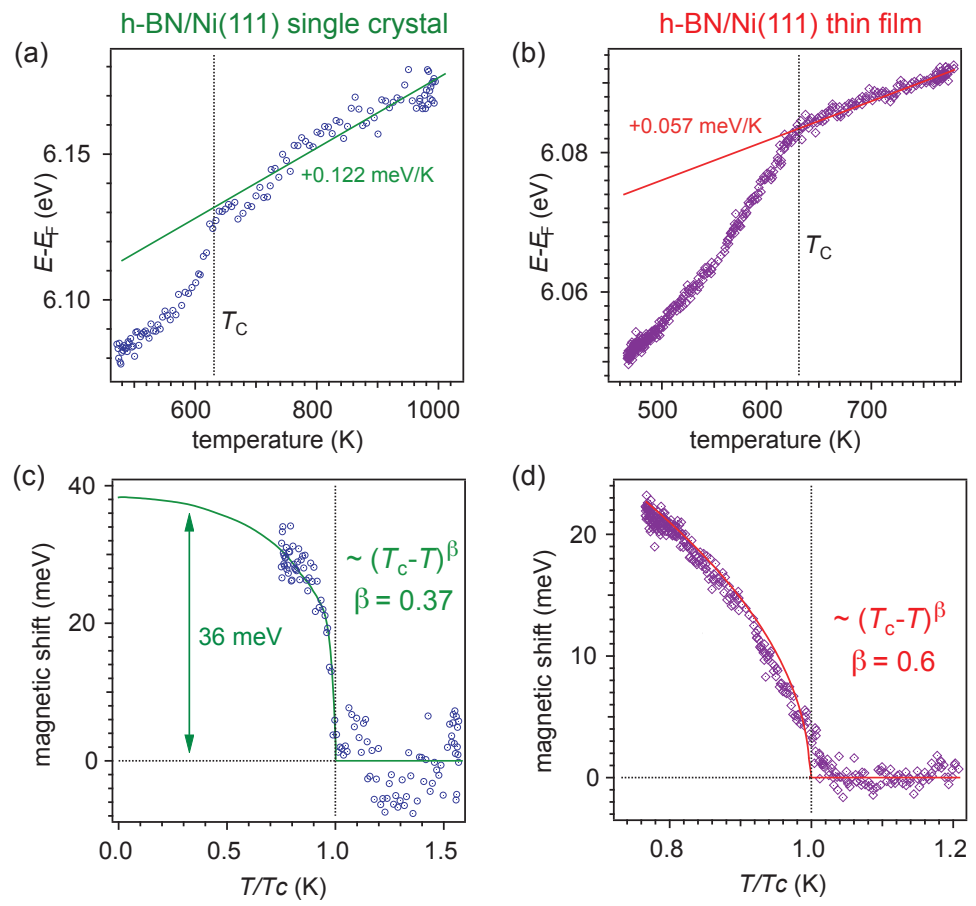

Figure 23. Study of induced exchange splitting of the IPS on $h-\mathrm{BN} / \mathrm{Ni}(111)$. Peak positions of the IPS as function of temperature for $h-\mathrm{BN}$ on a) bulk Ni and b) the $80 \mathrm{~nm} \mathrm{Ni(111)} \mathrm{film} \mathrm{[88].} \mathrm{The} \mathrm{lines} \mathrm{are} \mathrm{fits} \mathrm{to} \mathrm{the} \mathrm{linear} \mathrm{change} \mathrm{to} \mathrm{the} \mathrm{thermally}$ induced shift above the Curie temperature. c) and d) Peak shifts after subtraction of the thermal shift together with appropriate fits of the order parameter for bulk and thin film, respectively.

with $\beta=0.37$ for bulk nickel at $T \approx T_{c}$, as shown in the inset of Fig. 23 . In the case of the nickel film the fitted value $\beta=0.6$ is higher than in the bulk case. We conjecture that this might be due to the different magnetic anisotropy energy in the thin film sample because [111] direction corresponds to the magnetic easy axis of nickel [93].

The trend of the magnetic shift towards higher energy with increasing sample temperature suggests that the signal has majority character. Indeed preliminary spinresolved measurements at the COPHEE endstation at the Swiss Light Source [91], taking advantage of the strong $2 \mathrm{PPE}$ resonance in $h-\mathrm{BN} / \mathrm{Ni}(111)$ confirm the majority character of the photoelectron spin polarisation of the resonance peak [144], which has - as outlined in the preceding section - strong contributions from the IPS. We do not observe a second peak from minority owing to the fully polarised initial states, which were identified as $\mathrm{Ni} \Lambda_{3}$ states with majority spin close to the bulk L point of the BZ.

The second result from the fit is the expected total magnetic shift of the majority state. Since the minority state is not observed, the full exchange splitting can only be estimated assuming the double of the magnetic shift of the majority state. The latter is found by extrapolation of the magnetisation curve to $T=0$, what yields $36 \mathrm{meV}$, thus in the same order of magnitude than the splitting seen on $\mathrm{Ni}(111)$ [127]. The exchange splitting would then correspond to about $72 \mathrm{meV}$ at zero temperature, what is below 


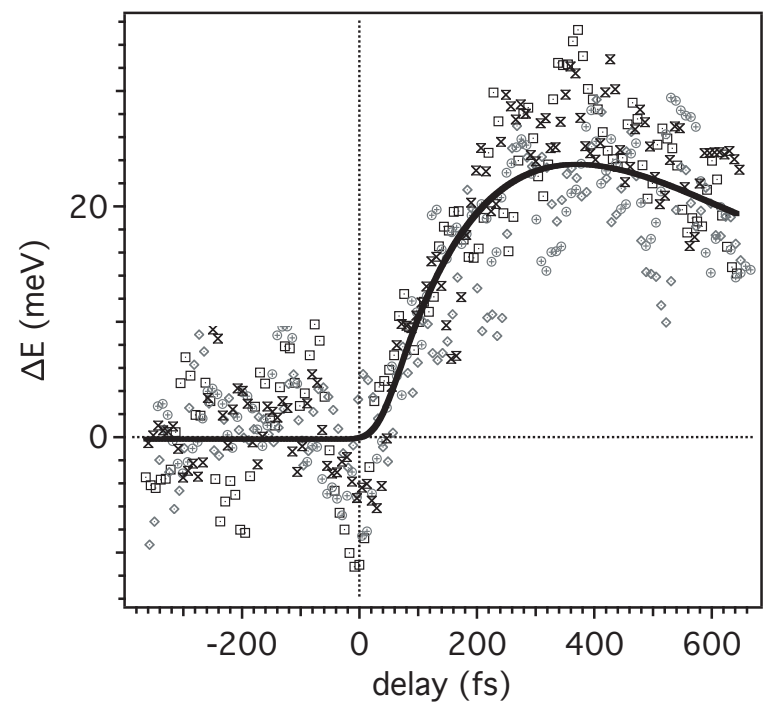

Figure 24. Ultrafast demagnetisation probed using the IPS in $h-\mathrm{BN} / \mathrm{Ni}(111)$. Energy shift of the IPS; the different symbols represent different data sets, the solid line is a simple bi-exponential fit with a rise time constant of $310 \mathrm{fs}$ and a decay constant of $820 \mathrm{fs}$. The average maximum shift of typically $28-32 \mathrm{meV}$ is observed at about $350 \mathrm{fs}$. The graphics are taken from Ref. [88].

the intrinsic linewidth of $84 \mathrm{meV}$ of the Ni(111) IPS as measured with high-resolution 2PPE [126]. This gives further evidence for a rather strong interaction of the IPS with the nickel substrate. For the $80 \mathrm{~nm}$ film sample we obtain a room temperature value of about $30 \mathrm{meV}$. These data thus demonstrate that the investigated IPS transition is sensitive to the ferromagnetic phase change in nickel and may therefore be used to probe laser pulse induced ultrafast demagnetisation of the nickel substrate.

\subsection{Ultrafast demagnetisation of nickel}

In 1996, Beaurepaire et al. reported on pioneering experiments on the magneto-optical behaviour of nickel thin films after pulsed laser irradiation [145]. Within the first picosecond after laser excitation, half of the magnetisation was lost. These results were later on confirmed in numerous studies including time-resolved photoemission experiments [146-148] and lead to a microscopic model applicable to solids [149]. In particular, it has been shown on $\mathrm{Ni}(111)$ by Rhie et al. using time-resolved ARPES that the magnetic exchange splitting between majority and minority spin $3 \mathrm{~d}$-bands in a $\mathrm{Ni}$ film collapses on a time scale of $\sim 300$ fs following a femtosecond laser pulse [147]. In a similar approach to that, we excited the $h-\mathrm{BN} / \mathrm{Ni}(111)$ sample by intense s-polarised infrared light at $1.55 \mathrm{eV}$ and probed the transient magnetic state on a femtosecond timescale by recording the spectra of the IPS [95].

The transient IPS energy as function of delay time after absorption of the pump pulse and after correction for pump-induced spectral shifts is shown in Fig. 24. We 
emphasise that thermal shifts due to lattice expansion like seen in static temperaturedependent experiments can be ruled out on such fast timescales of the order of $100 \mathrm{fs}$ [148]. The peak shift is interpreted as shift due to the collapse of the induced exchange splitting and, thereby, as an evidence for a laser pulse induced reduction of the magnetisation in $\mathrm{Ni}(111)$. The timescale of about 300 fs matches well the demagnetisation time observed in pristine $\mathrm{Ni}(111)$ [147]. Finally, based on a threetemperature model [145], which describes the magnetisation by a spin temperature $T_{s}$, a phenomenological model of energy exchange between the electron, spin, and phonon baths was derived. In this sense, the maximum shift observed in our experiment would correspond after calibration with Fig. 23 to a spin temperature close to $T_{s} \approx T_{c}$. As a conclusion, these findings show that magnetic interactions can be observed and even mediated on ultrafast timescales by free-electron like states across the $h$-BN-film, which could have broad impact on the development of miniature devices working at high, optical frequencies.

\section{Outlook: $h-\mathrm{BN} /$ graphene heterostructures and corrugated layers}

The hot carrier dynamics and energy dissipation within the $\pi$-bands of single-[150-152] and bilayer [153] graphene were subject of several studies by time-resolved ARPES (for a recent review see Ref. [154]). Carrier multiplication, i.e. the number increase of lowenergy charge carriers after excitation of a single electron-hole pair, is believed to be a promising route towards fast optoelectronic devices $[155,156]$. The ionisation caused by impact of electrons with sufficiently high energy to generate a number of electron-hole pairs is the key [157] and was shown to be the main channel determining the energy re-distribution and dissipation in graphene on insulating surfaces like sapphire [158] and $\mathrm{SiC}[152]$, for instance.

The opening of a gap at the Dirac point in graphene has direct implications on the dynamics of excited carriers in the $\pi$-bands $[153,155]$. As already mentioned in the introduction, $h-\mathrm{BN}$ constitutes a prime material for supporting while electronically isolating graphene. Heterostructures can be obtained by mechanical exfoliation of different layers and subsequent stacking what often leads to small patches with atomically clean interfaces and aggregates of contaminants [46]. An alternative way leading to chemically clean interfaces on larger length scales is to grow graphene in situ on $h$-BN. ARPES [159-161] and nano-ARPES (i.e. ARPES with a small, nanometersized light spot) measurements $[162,163]$ are available, as for example shown in Fig. 25 for graphene grown on a monolayer of $h$-BN/Cu(111) [159]. At the $\mathrm{K}$ both $\pi$-bands can clearly be distinguished. The $h-\mathrm{BN}$ layer retains the dispersion of the bare monolayer $h-\mathrm{BN} / \mathrm{Cu}(111)$ system. The Dirac point is found at $290 \mathrm{meV}$ below $E_{F}$ indicating electron doping through the $h$-BN-layer. Moreover, a close up view of the dispersion around $\overline{\mathrm{K}}$ reveals that momenta $k_{\|}$of the apex of the $h-\mathrm{BN} \pi$-band and of the Fermi level crossing of the graphene $\pi$-band do not coincide due to the slight lattice mismatch of the two hexagonal 2D networks. Graphene forms an incommensurate layer evidenced by 
a moiré pattern in low-energy-electron diffraction and scanning tunnelling microscopy images and, thereby, preserves its electronic properties, and no gap opens up at the Dirac point [159].
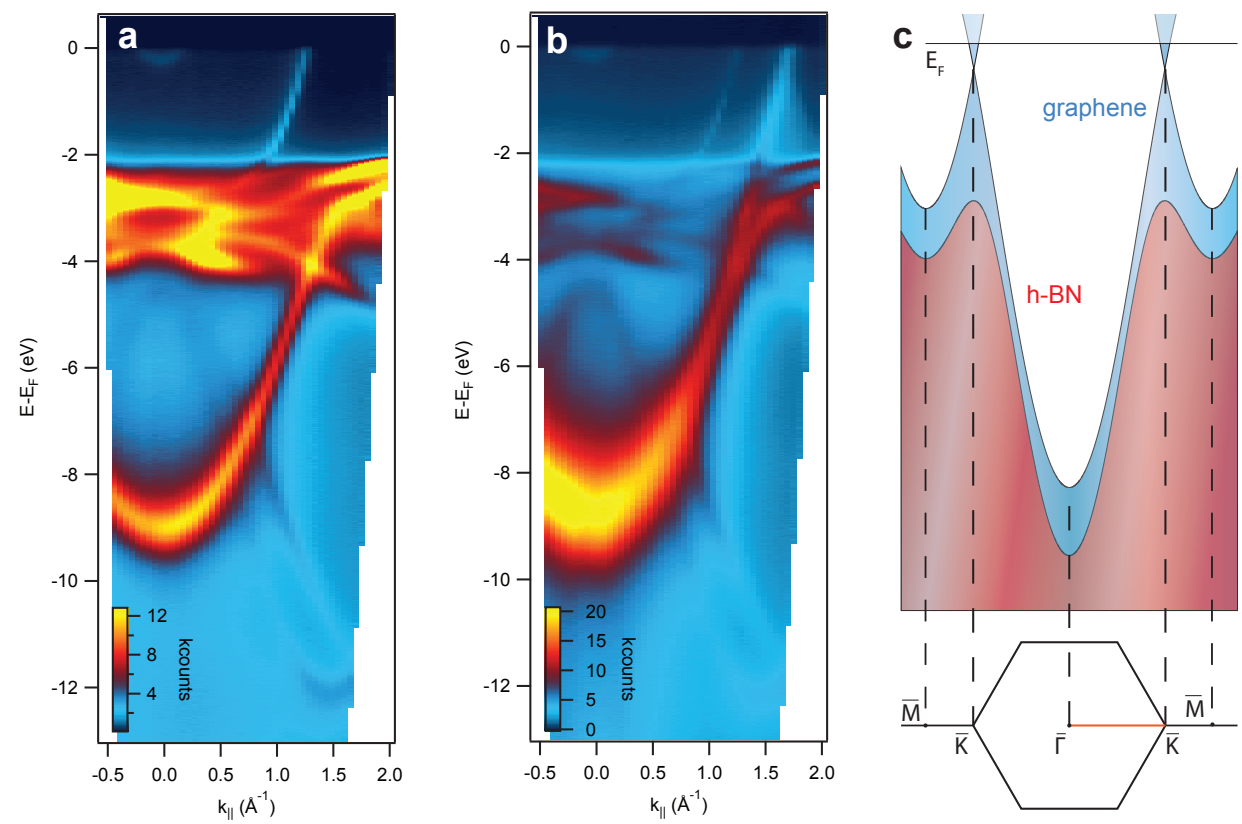

Figure 25. ARPES taken with $\mathrm{He} \mathrm{II} \alpha$ radiation $(40.8 \mathrm{eV})$ from a monolayer $\mathrm{g} / h_{-}$ BN heterostructure grown by sequential CVD on the $\mathrm{Cu}(111)$ surface. a) Bare $h^{-}$ $\mathrm{BN} / \mathrm{Cu}(111) ; \mathrm{b}) \mathrm{g} / h-\mathrm{BN} / \mathrm{Cu}(111)$. c) Sketch of the $\pi$-band dispersion along $\overline{\Gamma K}$ and surface BZ. Reprinted with permission from Ref. [159]. Copyright 2013 American Chemical Society.

The workfunction of this heterostructure is about $4.25 \mathrm{eV}$. If we are to look for interface and image potential states at similar energies as for $\mathrm{Ni}(111)$, we must use photon energies around $2 \mathrm{eV}$. As a consequence, the spectra are dominated by threephoton transitions. Such spectra are shown in Fig. 26. A clear resonance can be seen at $h \nu \approx 2 \mathrm{eV}$. Since the possible initial states must be located in the structureless sp-bulk bands of $\mathrm{Cu}$ (see Fig. 26b), we probably deal with a resonant transition involving two intermediate states which are separated by $2 \mathrm{eV}$. The peak moves with once the photon energy and is, thereby, determined by the energy of the second intermediate state in this three-photon process. The resonance energy translates into an intermediate state energy of $3.37 \mathrm{eV}$ above $E_{F}$, thus $0.88 \mathrm{eV}$ below the vacuum level $E_{\text {vac. }}$. Within the error bars of our measurements this corresponds to the maximum binding energy of a $n=1$ image potential state in front of a perfect metal [116]. Again subtracting the resonance photon energy, we find the first intermediate state at around $1.4 \mathrm{eV}$ above the Fermi level, thus roughly in the same energy range as for $h-\mathrm{BN} / \mathrm{Ni}(111)$.

Besides epitaxial layers on surfaces like $\mathrm{Ni}(111)$ or $\mathrm{Cu}(111)$, monolayer $h-\mathrm{BN}$ can form strongly corrugated networks when adsorbed on surfaces with large lattice mismatch like $\mathrm{Rh}(111)$ [64] or $\mathrm{Ru}(0001)$ [58]. These superstructures were termed 

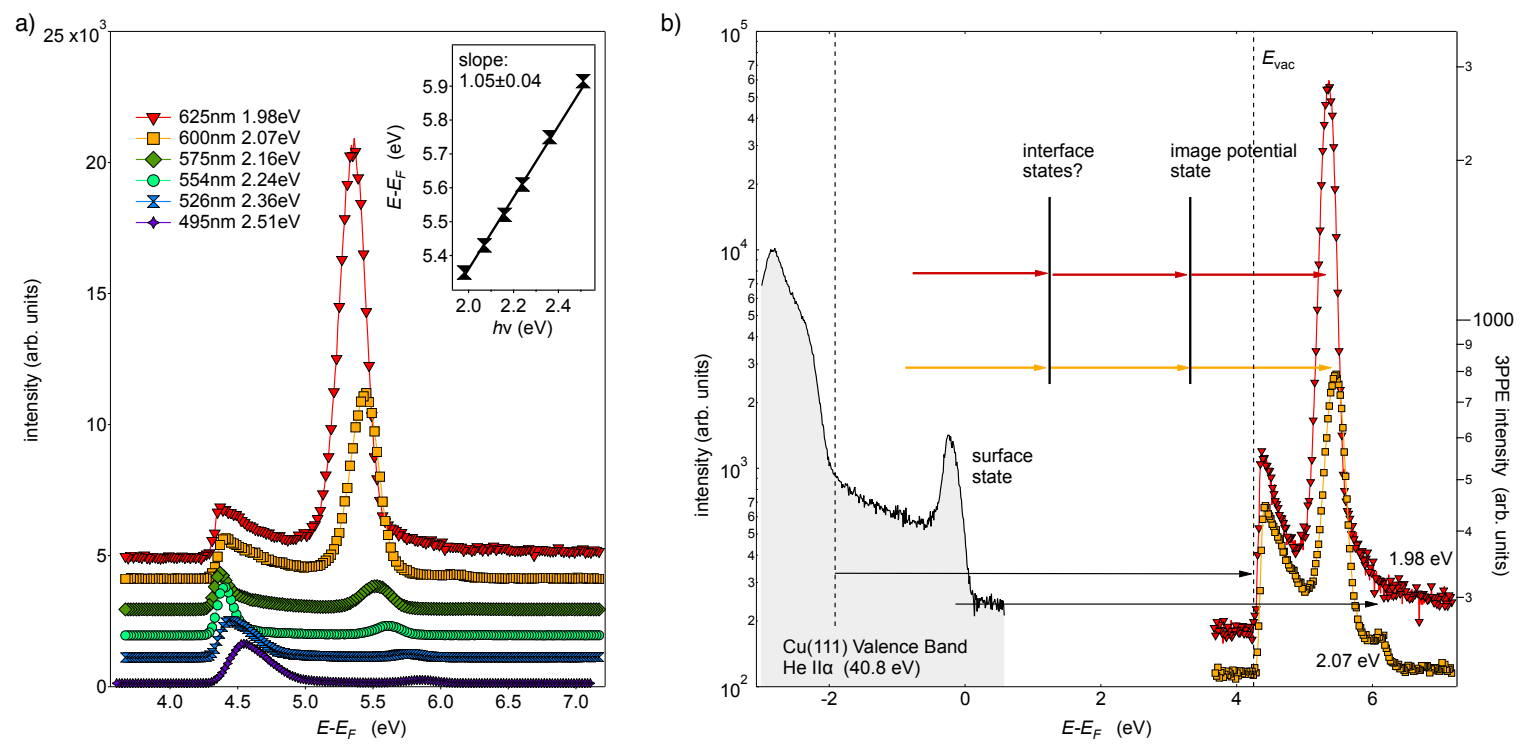

Figure 26. Three-photon photoemission (3PPE) from the $\mathrm{g} / h-\mathrm{BN} / \mathrm{Cu}(111)$ heterostructure of Fig. 25. a) Spectra for several photon energies. Inset: Peak energies plotted as function of photon energy together with a line fit. b) Scheme of possible transitions for two photon energies on resonance $(h \nu=1.98 \mathrm{eV}$ [red symbols], $2.07 \mathrm{eV}$ [yellow symbols]), out of the $\mathrm{Cu}(111)$ sp-valence band via two intermediate states. The two black arrows indicate three-photon transitions arriving at the vacuum cutoff $E_{\mathrm{vac}}$ and out of the $\mathrm{Cu}(111)$ surface state. The latter shows up as small peak in the $2.07 \mathrm{eV}$ spectrum.

nanomesh and can be used, if not for their own properties, as templates for building molecular array structures $[11,101]$. As a consequence of the structural corrugation in nanomesh-like $h-\mathrm{BN}$, valence energy bands are split [64]. There are first indications in preliminary two- and three-photon photoemission experiments that the conduction bands are split, and in particular that interface states exist and are split with similar intensity ratio than corresponding valence band peaks [88]. These states sense the lateral potential landscape as the probability distribution of the wavefunction is different at $\mathrm{N}$ and B sites [73]. This would be in line with Xe desorption experiments in which is was shown that the structural corrugation of the $h$-BN lattice is directly imprinted into the potential landscape and leads to strong and sizeable in-plane fields [12].

Moreover, in an attempt to grow graphene on $h-\mathrm{BN}$ nanomesh on $\mathrm{Rh}(111)$, Roth and coworkers could show that the typical ARPES features like Dirac cones out of $\pi$ states appear only if the $h$-BN-layer is flattened by preceding intercalation (of carbon) followed by annealing [160]. In turn, in early NEXAFS experiments, Preobrajenski et al. demonstrated by controlled generation of defects in $h-\mathrm{BN} / \mathrm{Ni}(111)$ that the signal from the interface state disappears with increasing defect density [122]. It will be interesting to study the effects of such intercalation studies on the presence and lifetimes of interface states and to relate them to the quality and finally transport properties of the graphene flakes grown in this way on highly stable substrates like nanomesh. 


\section{Conclusion}

We are aware that this paper might rather open up new questions than giving definite answers. $h-\mathrm{BN}$, graphene and systems combined of both are topic of intensive current research efforts. It remains puzzling that the $\pi^{\star}$-bands dominate the electronic and optical response in monolayer and bulk $h-\mathrm{BN}$ to high-energy electron impact $[29,39]$ or optical stimuli in the ultraviolet [33] and x-ray range [122] but that the $\pi^{\star}$-band was never directly observed with momentum-resolved probes to the best of our knowledge. It will be very instructive to see the dispersion and lifetime of electrons excited into these states which so far remained inaccessible for all kinds of different wavelength and polarisation combinations for pump and probe pulses [164]. Nevertheless, it could be demonstrated that and how the $h-\mathrm{BN}$-derived states depend on dielectric and magnetic properties of the metal substrate, what opens up new ways for creating tailored interfaces by sequential CVD processes.

We hope that this works stimulates further theoretical and experimental efforts for both, single layer and bulk systems. Interlayer states were often ignored in theory and analysis of experimental data. We find that the dynamic effects are very strong and surprising. Moreover, due to the fact that the charge density is mostly located outside the atomic planes, and that these states exist for single layers and bulk $h$-BNas well as for graphene and graphite, they could play a major role for the properties of heretostacks by facilitating charge transfer across the interlayer gaps. Even in close contact to an open-d-shell metal like nickel, these states possess surprisingly long lifetimes of the order of one hundred femtoseconds, probably due to excitonic effects stabilising the electronhole pair excitations.

On the theoretical side, since the presence of these interlayer or interface states was shown to shuffle bands in the unoccupied bandstructure, they have to be included in any calculation, even if they are not expected to contribute significantly to in-plane carrier transport or exciton dynamics. In the case of $h$-BN monolayers on metal surfaces, image potential states exist outside the surface. They behave similarly to the NFE interlayer states with free-electron-like dispersion and very long lifetimes. It is surprising that the interaction of these states with the substrate is strong enough to induce an exchange splitting even across the $h$-BN layer. This can only be explained by spin-dependent reflection at the substrate surface meaning that the electron are reflected back and forth at the metal surface and the image potential outside [117]. We propose that the $h-\mathrm{BN}$ layer acts like a dielectric continuum [116], which produces a small potential step due to the out-of-plane dipole moment but stays transparent for these electrons otherwise. This might have implications for the use of $h-\mathrm{BN}$ as dielectric for voltage gating or simply as insulating spacer in thin-film electronics. 


\section{Acknowledgments}

Over the years many people contributed to these measurements: we had valuable discussions with Willi Auwärter, Martina Corso, Simon Berner, Thomas Brugger, Hugo Dil, Markus Donath, François Ducastelle and Annick Loiseau, and in particular with Thomas Greber and Jürg Osterwalder. Andrei Dolocan, and Hansjörg Neff, and Jorge Lobo-Checa helped with the experimental setup and taking measurements. Thomas Ruchti and Wolfgang Schüsslbauer (formerly Time-Bandwidth-Products) kindly provided a laser setup for taking spin-resolved 2PPE spectra. We acknowledge Stefan Gsell and Matthias Schreck (U Augsburg) for providing Ni/sapphire films for the demagnetisation experiments. One of us, M.H. acknowledges the hospitality of the group of Markus Donath at the University of Münster, and the Research Grant of the University of Zurich for financial support. This work was funded by the Swiss National Science Foundation.

\section{References}

[1] Novoselov K S, Geim A K, Morozov S V, Jiang D, Zhang Y, Dubonos S V, Grigorieva I V and Firsov A A 2004 Science 306666 URL http://science. sciencemag. org/content/306/5696/666. abstract

[2] Geim A K and Novoselov K S 2007 Nature Materials 6183 EP - URL https: //doi.org/10.1038/nmat1849

[3] Zhang Y, Rubio A and Lay G L 2017 Journal of Physics D: Applied Physics 50 053004 URL http://dx.doi.org/10.1088/1361-6463/aa4e8b

[4] Xiao D, Liu G B, Feng W, Xu X and Yao W 2012 Physical Review Letters 108 196802- URL https://link.aps.org/doi/10.1103/PhysRevLett.108.196802

[5] Mak K F, He K, Shan J and Heinz T F 2012 Nature Nanotechnology 7494 EP URL https://doi.org/10.1038/nnano.2012.96

[6] Cao T, Wang G, Han W, Ye H, Zhu C, Shi J, Niu Q, Tan P, Wang E, Liu B and Feng J 2012 Nature Communications 3887 EP - URL https://doi.org/ $10.1038 /$ ncomms 1882

[7] Xiao D, Yao W and Niu Q 2007 Physical Review Letters 99 236809- URL https://link.aps.org/doi/10.1103/PhysRevLett.99.236809

[8] Rycerz A, Tworzydło J and Beenakker C W J 2007 Nature Physics 3172 EP URL https://doi.org/10.1038/nphys547

[9] Siria A, Poncharal P, Biance A L, Fulcrand R, Blase X, Purcell S T and Bocquet L 2013 Nature 494455 EP - URL https://doi.org/10.1038/nature11876

[10] Cun H, Iannuzzi M, Hemmi A, Osterwalder J and Greber T 2014 ACS Nano 8 7423-7431 URL https://doi.org/10.1021/nn502645w 
[11] Berner S, Corso M, Widmer R, Groening O, Laskowski R, Blaha P, Schwarz K, Goriachko A, Over H, Gsell S, Schreck M, Sachdev H, Greber T and Osterwalder J 2007 Angewandte Chemie International Edition 46 5115-5119 URL https: //doi.org/10.1002/anie.200700234

[12] Dil H, Lobo-Checa J, Laskowski R, Blaha P, Berner S, Osterwalder J and Greber T 2008 Science 3191824 URL http://science.sciencemag.org/content/319/ $5871 / 1824$.abstract

[13] Brugger T, Günther S, Wang B, Dil J H, Bocquet M L, Osterwalder J, Wintterlin J and Greber T 2009 Physical Review B 79 045407-URL https://link.aps. org/doi/10.1103/PhysRevB.79.045407

[14] Mertens S F L, Hemmi A, Muff S, Gröning O, De Feyter S, Osterwalder J and Greber T 2016 Nature 534676 EP - URL https://doi.org/10.1038/ nature 18275

[15] Logan B E and Elimelech M 2012 Nature 488313 EP - URL https://doi.org/ $10.1038 /$ nature 11477

[16] Cun H, Hemmi A, Miniussi E, Bernard C, Probst B, Liu K, Alexander D T L, Kleibert A, Mette G, Weinl M, Schreck M, Osterwalder J, Radenovic A and Greber T 2018 Nano Letters 18 1205-1212 URL https://doi.org/10.1021/ acs.nanolett.7b04752

[17] Cho Y J, Summerfield A, Davies A, Cheng T S, Smith E F, Mellor C J, Khlobystov A N, Foxon C T, Eaves L, Beton P H and Novikov S V 2016 Scientific Reports 6 34474 EP - URL https://doi.org/10.1038/srep34474

[18] Mishchenko A, Tu J S, Cao Y, Gorbachev R V, Wallbank J R, Greenaway M T, Morozov V E, Morozov S V, Zhu M J, Wong S L, Withers F, Woods C R, Kim Y J, Watanabe K, Taniguchi T, Vdovin E E, Makarovsky O, Fromhold T M, Fal'ko V I, Geim A K, Eaves L and Novoselov K S 2014 Nature Nanotechnology 9808 EP - URL https://doi.org/10.1038/nnano.2014.187

[19] Vdovin E E, Mishchenko A, Greenaway M T, Zhu M J, Ghazaryan D, Misra A, Cao Y, Morozov S V, Makarovsky O, Fromhold T M, Patanè A, Slotman G J, Katsnelson M I, Geim A K, Novoselov K S and Eaves L 2016 Physical Review Letters 116 186603-URL https://link.aps.org/doi/10.1103/PhysRevLett. 116.186603

[20] Unuchek D, Ciarrocchi A, Avsar A, Watanabe K, Taniguchi T and Kis A 2018 Nature 560 340-344 URL https://doi.org/10.1038/s41586-018-0357-y

[21] Ciarrocchi A, Unuchek D, Avsar A, Watanabe K, Taniguchi T and Kis A 2019 Nature Photonics 13 131-136 URL https://doi.org/10.1038/ s41566-018-0325-y

[22] Zasada I, Maślanka P, Molenda A and Łuczak K 2018 Journal of Physics: Condensed Matter 30055501 URL http://dx.doi.org/10.1088/1361-648X/ aa9fd9 
[23] Molenda A, Zasada I and Maślanka P 2019 Physica E: Low-dimensional Systems and Nanostructures 107 160-169 URL http://www.sciencedirect. com/science/article/pii/S1386947718315169

[24] Cadiz F, Courtade E, Robert C, Wang G, Shen Y, Cai H, Taniguchi T, Watanabe K, Carrere H, Lagarde D, Manca M, Amand T, Renucci P, Tongay S, Marie X and Urbaszek B 2017 Physical Review X 7 021026- URL https://link.aps. org/doi/10.1103/PhysRevX.7.021026

[25] Liu S, Yuan K, Xu X, Yin R, Lin D Y, Li Y, Watanabe K, Taniguchi T, Meng Y, Dai L and Ye Y 2019 Advanced Electronic Materials 51800419 URL https://doi.org/10.1002/aelm.201800419

[26] Han X, Lin J, Liu J, Wang N and Pan D 2019 The Journal of Physical Chemistry C 123 14797-14802 URL https://doi.org/10.1021/acs.jpcc.9b02549

[27] Catellani A, Posternak M, Baldereschi A, Jansen H J F and Freeman A J 1985 Physical Review B 32 6997-6999 URL https://link.aps.org/doi/10.1103/ PhysRevB.32.6997

[28] Blase X, Rubio A, Louie S G and Cohen M L 1995 Physical Review B 516868 6875 URL https://link.aps.org/doi/10.1103/PhysRevB.51.6868

[29] Watanabe K, Taniguchi T and Kanda H 2004 Nature Materials 3 404-409 URL https://doi.org/10.1038/nmat1134

[30] Arnaud B, Lebègue S, Rabiller P and Alouani M 2006 Physical Review Letters 96 026402-URL https://link.aps.org/doi/10.1103/PhysRevLett.96.026402

[31] Wirtz L, Marini A, Grüning M, Attaccalite C, Kresse G and Rubio A 2008 Physical Review Letters 100 189701- URL https://link.aps.org/doi/10. 1103/PhysRevLett.100.189701

[32] Arnaud B, Lebègue S, Rabiller P and Alouani M 2008 Physical Review Letters 100 189702-URL https://link.aps.org/doi/10.1103/PhysRevLett.100.189702

[33] Cassabois G, Valvin P and Gil B 2016 Nature Photonics 10262 EP - URL https://doi.org/10.1038/nphoton.2015.277

[34] Kubota Y, Watanabe K, Tsuda O and Taniguchi T 2007 Science 317932 URL http://science.sciencemag. org/content/317/5840/932. abstract

[35] Dahal R, Li J, Majety S, Pantha B N, Cao X K, Lin J Y and Jiang H X 2011 Applied Physics Letters 98211110 URL https://doi.org/10.1063/1.3593958

[36] Watanabe K, Taniguchi T, Niiyama T, Miya K and Taniguchi M 2009 Nature Photonics 3591 EP - URL https://doi.org/10.1038/nphoton.2009.167

[37] Schué L, Sponza L, Plaud A, Bensalah H, Watanabe K, Taniguchi T, Ducastelle F, Loiseau A and Barjon J 2019 Physical Review Letters 122 067401- URL https://link.aps.org/doi/10.1103/PhysRevLett.122.067401

[38] Morimoto H, Hazama Y, Tanaka K and Naka N 2016 Diamond and Related Materials 63 47-50 URL http://www.sciencedirect.com/science/article/ pii/S0925963515300893 
[39] Watanabe K and Taniguchi T 2011 International Journal of Applied Ceramic Technology 8 977-989 URL https://doi.org/10.1111/j.1744-7402.2011. 02626.x

[40] Teke A, Özgür Ü, Doğan S, Gu X, Morkoç H, Nemeth B, Nause J and Everitt H O 2004 Physical Review B 70 195207-URL https://link.aps.org/doi/10. 1103/PhysRevB.70.195207

[41] Schué L, Berini B, Betz A C, Plaçais B, Ducastelle F, Barjon J and Loiseau A 2016 Nanoscale 8 6986-6993 URL http://dx.doi.org/10.1039/C6NR01253A

[42] Sponza L, Amara H, Attaccalite C, Latil S, Galvani T, Paleari F, Wirtz L and Ducastelle F 2018 Physical Review B 98 125206-URL https://link.aps.org/ doi/10.1103/PhysRevB.98.125206

[43] Schué L, Stenger I, Fossard F, Loiseau A and Barjon J 2016 2D Materials 4015028 URL http://dx.doi.org/10.1088/2053-1583/4/1/015028

[44] Gorbachev R V, Riaz I, Nair R R, Jalil R, Britnell L, Belle B D, Hill E W, Novoselov K S, Watanabe K, Taniguchi T, Geim A K and Blake P 2011 Small 7 465-468 URL https://doi.org/10.1002/smll.201001628

[45] Levendorf M P, Kim C J, Brown L, Huang P Y, Havener R W, Muller D A and Park J 2012 Nature 488627 EP - URL https ://doi .org/10.1038/nature11408

[46] Haigh S J, Gholinia A, Jalil R, Romani S, Britnell L, Elias D C, Novoselov K S, Ponomarenko L A, Geim A K and Gorbachev R 2012 Nature Materials 11 764-767 URL https://doi.org/10.1038/nmat3386

[47] Lee K H, Shin H J, Lee J, Lee I y, Kim G H, Choi J Y and Kim S W 2012 Nano Letters 12 714-718 URL https://doi.org/10.1021/nl203635v

[48] Vangala S, Siegel G, Prusnick T and Snure M 2018 Scientific Reports 88842 URL https : //doi.org/10.1038/s41598-018-27237-z

[49] Dean C R, Young A F, Meric I, Lee C, Wang L, Sorgenfrei S, Watanabe K, Taniguchi T, Kim P, Shepard K L and Hone J 2010 Nature Nanotechnology 5722 EP - URL https://doi.org/10.1038/nnano.2010.172

[50] Yang W, Berthou S, Lu X, Wilmart Q, Denis A, Rosticher M, Taniguchi T, Watanabe K, Fève G, Berroir J M, Zhang G, Voisin C, Baudin E and Plaçais B 2018 Nature Nanotechnology 13 47-52 URL https://doi.org/10. 1038/s41565-017-0007-9

[51] Wang J, Ma F, Liang W and Sun M 2017 Materials Today Physics 2 6-34 URL http://www.sciencedirect.com/science/article/pii/S2542529317300597

[52] Hüfner S 1995 Photoelectron Spectroscopy, Principles and Applications 1st ed (Springer Series in Solid-State Sciences vol 82) (Springer, Berlin, Heidelberg) URL https://doi.org/10.1007/978-3-662-03150-6

[53] Nagashima A, Tejima N, Gamou Y, Kawai T and Oshima C 1995 Physical Review Letters 75 3918-3921 URL https ://link .aps.org/doi/10.1103/PhysRevLett. 75.3918 
[54] Nagashima A, Gamou Y, Terai M, Wakabayashi M and Oshima C 1996 Physical Review B 54 13491-13494 URL https://link.aps.org/doi/10.1103/ PhysRevB.54.13491

[55] Rokuta E, Hasegawa Y, Suzuki K, Gamou Y, Oshima C and Nagashima A 1997 Physical Review Letters 79 4609-4612 URL https://link.aps.org/doi/10. 1103/PhysRevLett. 79.4609

[56] Morscher M, Corso M, Greber T and Osterwalder J 2006 Surface Science 600 3280-3284 URL http://www.sciencedirect.com/science/article/pii/ S0039602806007266

[57] Allan M P, Berner S, Corso M, Greber T and Osterwalder J 2007 Nanoscale Research Letters 294 URL https://doi.org/10.1007/s11671-006-9036-2

[58] Goriachko A, He, Knapp M, Over H, Corso M, Brugger T, Berner S, Osterwalder J and Greber T 2007 Langmuir 23 2928-2931 URL https://doi.org/10.1021/ la062990t

[59] Martoccia D, Brugger T, Björck M, Schlepütz C M, Pauli S A, Greber T, Patterson B D and Willmott P R 2010 Surface Science 604 L16-L19 URL http://www.sciencedirect.com/science/article/pii/S0039602810000051

[60] Hutchings G S, Jhang J H, Zhou C, Hynek D, Schwarz U D and Altman E I 2017 ACS Applied Materials \& Interfaces 9 11266-11271 URL https://doi.org/10. 1021/acsami.7b01369

[61] Stania R, Heckel W, Kalichava I, Bernard C, Kerscher T C, Cun H Y, Willmott P R, Schönfeld B, Osterwalder J, Müller S and Greber T 2016 Physical Review B 93 161402-URL https://link.aps.org/doi/10.1103/PhysRevB.93.161402

[62] Yin J, Liu X, Lu W, Li J, Cao Y, Li Y, Xu Y, Li X, Zhou J, Jin C and Guo W 2015 Small 11 5375-5380 URL https://doi.org/10.1002/smll.201501439

[63] Li X, Sundaram S, El Gmili Y, Ayari T, Puybaret R, Patriarche G, Voss P L, Salvestrini J P and Ougazzaden A 2016 Crystal Growth \& Design 16 3409-3415 URL https://doi.org/10.1021/acs.cgd.6b00398

[64] Corso M, Auwärter W, Muntwiler M, Tamai A, Greber T and Osterwalder J 2004 Science 303217 URL http://science.sciencemag.org/content/303/5655/ 217. abstract

[65] Laskowski R, Blaha P and Schwarz K 2008 Physical Review B 78 045409- URL https://link.aps.org/doi/10.1103/PhysRevB.78.045409

[66] Preobrajenski A B, Nesterov M A, Ng M L, Vinogradov A S and Mårtensson N 2007 Chemical Physics Letters 446 119-123 URL http://www. sciencedirect. com/science/article/pii/S0009261407010998

[67] Auwärter W 2019 Surface Science Reports 74 1-95 URL http://www . sciencedirect.com/science/article/pii/S0167572918300517

[68] Oshima C and Nagashima A 1997 Journal of Physics: Condensed Matter 9 1-20 URL http://dx.doi.org/10.1088/0953-8984/9/1/004 
[69] Greber T 2010 Graphene and boron nitride single layers Handbook of Nanophysics: Functional Nanomaterials ed Sattler K (CRC Press, Boca Raton) chap 18 1st ed URL https://www.crcpress.com/ Handbook-of-Nanophysics-Functional-Nanomaterials/Sattler/p/book/ 9781138111936

[70] Nai C T, Lu J, Zhang K and Loh K P 2015 The Journal of Physical Chemistry Letters 6 4189-4193 URL https://doi.org/10.1021/acs.jpclett.5b01900

[71] Paleari F, Galvani T, Amara H, Ducastelle F, Molina-Sánchez A and Wirtz L 2018 2D Materials 5045017 URL http://dx.doi.org/10.1088/2053-1583/aad586

[72] Tisdale W A, Muntwiler M, Norris D J, Aydil E S and Zhu X Y 2008 The Journal of Physical Chemistry C 112 14682-14692 URL https://doi.org/10. 1021/jp802455p

[73] Catellani A, Posternak M, Baldereschi A and Freeman A J 1987 Physical Review B 36 6105-6111 URL https://link.aps.org/doi/10.1103/PhysRevB.36.6105

[74] Dose V 1977 Applied physics 14 117-118 URL https://doi.org/10.1007/ BF00882639

[75] Himpsel F J and Fauster T 1984 Journal of Vacuum Science E Technology A 2 815-821 URL https://doi.org/10.1116/1.572514

[76] Smith N V 1988 Reports on Progress in Physics 51 1227-1294 URL http: //dx.doi.org/10.1088/0034-4885/51/9/003

[77] Donath M, Dose V, Ertl K and Kolac U 1990 Physical Review B 41 5509-5518 URL https://link.aps.org/doi/10.1103/PhysRevB.41.5509

[78] Steinmann W 1989 Applied Physics A 49 365-377 URL https://doi.org/10. $1007 / \mathrm{BF} 00615019$

[79] Hengsberger M, Baumberger F, Neff H J, Greber T and Osterwalder J 2008 Physical Review B 77 085425- URL https://link.aps.org/doi/10.1103/ PhysRevB.77.085425

[80] Haight R, Bokor J, Stark J, Storz R H, Freeman R R and Bucksbaum P H 1985 Physical Review Letters 54 1302-1305 URL https://link.aps.org/doi/ 10.1103/PhysRevLett.54.1302

[81] Hertel T, Knoesel E, Wolf M and Ertl G 1996 Physical Review Letters 76 535-538 URL https://link.aps.org/doi/10.1103/PhysRevLett.76.535

[82] Bauer M and Aeschlimann M 2002 Journal of Electron Spectroscopy and Related Phenomena 124 225-243 URL http://www.sciencedirect.com/science/ article/pii/S0368204802000567

[83] Giesen K, Hage F, Himpsel F J, Riess H J and Steinmann W 1985 Physical Review Letters 55 300-303 URL https://link.aps.org/doi/10.1103/PhysRevLett. 55.300

[84] Bokor J 1989 Science 2461130 URL http://science.sciencemag.org/ content/246/4934/1130. abstract 
[85] Wolf M, Hotzel A, Knoesel E and Velic D 1999 Physical Review B 59 5926-5935 URL https://link.aps.org/doi/10.1103/PhysRevB.59.5926

[86] Boger K, Roth M, Weinelt M, Fauster T and Reinhard P G 2002 Physical Review B 65 075104-URL https://link.aps.org/doi/10.1103/PhysRevB.65.075104

[87] Greber T, Raetzo O, Kreutz T J, Schwaller P, Deichmann W, Wetli E and Osterwalder J 1997 Review of Scientific Instruments 68 4549-4554 URL https: //doi.org/10.1063/1.1148429

[88] Leuenberger D 2011 Electron dynamics in low-dimensional surfaces studied by time-resolved photoelectron spectroscopy Ph.D. thesis University of Zurich URL http: //www.dissertationen.uzh.ch/

[89] Monney C, Schuler A, Jaouen T, Mottas M L, Wolf T, Merz M, Muntwiler M, Castiglioni L, Aebi P, Weber F and Hengsberger M 2018 Physical Review B 97 075110-URL https://link.aps.org/doi/10.1103/PhysRevB .97.075110

[90] Schuler A 2018 Design and Implementation of a Monochromatic High Harmonic Generation Light Source at $100 \mathrm{kHz}$ and its Application to Time- and AngleResolved Photoelectron Spectroscopy on $\mathrm{Bi}_{2} \mathrm{Se}_{3}$ Ph.D. thesis University of Zurich URL http://www.dissertationen.uzh.ch/

[91] Hoesch M, Greber T, Petrov V N, Muntwiler M, Hengsberger M, Auwärter W and Osterwalder J 2002 Journal of Electron Spectroscopy and Related Phenomena 124 263-279 URL http://www.sciencedirect.com/science/article/pii/ S0368204802000580

[92] Zumbrägel K, Wulff K, Eibl C, Donath M and Hengsberger M 2008 Physical Review B 78 085422- URL https : //link.aps .org/doi/10.1103/PhysRevB. 78. 085422

[93] Donath M 1994 Surface Science Reports 20 251-316 URL http://www. sciencedirect.com/science/article/pii/0167572994900108

[94] Muntwiler M, Hengsberger M, Dolocan A, Neff H, Greber T and Osterwalder J 2007 Physical Review B 75 075407-URL https://link.aps.org/doi/10.1103/ PhysRevB.75.075407

[95] Leuenberger D, Yanagisawa H, Roth S, Osterwalder J and Hengsberger M 2011 Physical Review B 84 125107-URL https://link.aps.org/doi/10.1103/ PhysRevB .84.125107

[96] Leuenberger D, Yanagisawa H, Roth S, Dil J H, Wells J W, Hofmann P, Osterwalder J and Hengsberger M 2013 Physical Review Letters 110136806 URL https://link.aps.org/doi/10.1103/PhysRevLett.110.136806

[97] Roth S, Leuenberger D, Osterwalder J, Dahl J E, Carlson R M K, Tkachenko B A, Fokin A A, Schreiner P R and Hengsberger M 2010 Chemical Physics Letters 495 102-108 URL http: //www.sciencedirect.com/science/article/ $\mathrm{pii/S0009261410008729}$ 
[98] Schuler A, Monney C, Grad L, Osterwalder J and Hengsberger M 2019 Design and commissioning of an high-repetition rate time- and angle-resolved photoemission experiment manuscript in preparation

[99] Auwärter W, Kreutz T J, Greber T and Osterwalder J 1999 Surface Science 429 229-236 URL http://www.sciencedirect.com/science/article/pii/ S0039602899003817

[100] Bunk O, Corso M, Martoccia D, Herger R, Willmott P R, Patterson B D, Osterwalder J, van der Veen J F and Greber T 2007 Surface Science 601 L7-L10 URL http://www.sciencedirect.com/science/article/ $\mathrm{pii/S0039602806011551}$

[101] Goriachko A and Over H 2009 Zeitschrift für Physikalische Chemie 223157 URL https://www.degruyter.com/view/j/zpch.2009.223.issue-1-2/zpch. 2009.6030/zpch.2009.6030.xml

[102] Grad G B, Blaha P, Schwarz K, Auwärter W and Greber T 2003 Physical Review B 68 085404-URL https://link.aps.org/doi/10.1103/PhysRevB.68.085404

[103] Auwärter W 2003 One Monolayer of Hexagonal Boron Nitride on Ni(111): an Atomically Sharp Interface Ph.D. thesis University of Zurich URL http://www. dissertationen.uzh.ch/

[104] Muntwiler M, Auwärter W, Baumberger F, Hoesch M, Greber T and Osterwalder J 2001 Surface Science 472 125-132 URL http://www.sciencedirect.com/ science/article/pii/S0039602800009286

[105] Gómez Díaz J, Ding Y, Koitz R, Seitsonen A P, Iannuzzi M and Hutter J 2013 Theoretical Chemistry Accounts 1321350 URL https://doi.org/10.1007/ s00214-013-1350-z

[106] Robertson J 1984 Physical Review B 29 2131-2137 URL https://link.aps.org/ doi/10.1103/PhysRevB.29.2131

[107] Wirtz L, Marini A and Rubio A 2006 Physical Review Letters 96 126104- URL https://link.aps.org/doi/10.1103/PhysRevLett.96.126104

[108] Fugallo G, Aramini M, Koskelo J, Watanabe K, Taniguchi T, Hakala M, Huotari S, Gatti M and Sottile F 2015 Physical Review B 92 165122- URL https: //link.aps.org/doi/10.1103/PhysRevB.92.165122

[109] Galvani T, Paleari F, Miranda H P C, Molina-Sánchez A, Wirtz L, Latil S, Amara H and Ducastelle F 2016 Physical Review B 94 125303- URL https: //link.aps.org/doi/10.1103/PhysRevB.94.125303

[110] Giraud P 2012 Study of the Electronic Structure of hexagonal Boron Nitride on metals substrates Master's thesis Université des Sciences et Technologies Lille 1 URL http://nano-bio.ehu.es/thesis/master

[111] Wallace P R 1947 Physical Review 71 622-634 URL https://link.aps.org/ doi/10.1103/PhysRev.71.622 
[112] Henck H, Pierucci D, Fugallo G, Avila J, Cassabois G, Dappe Y J, Silly M G, Chen C, Gil B, Gatti M, Sottile F, Sirotti F, Asensio M C and Ouerghi A 2017 Physical Review B 95 085410-URL https://link.aps .org/doi/10.1103/PhysRevB. 95. 085410

[113] Huda M N and Kleinman L 2006 Physical Review B 74 075418- URL https: //link.aps.org/doi/10.1103/PhysRevB.74.075418

[114] Posternak M, Baldereschi A, Freeman A J, Wimmer E and Weinert M 1983 Physical Review Letters 50 761-764 URL https://link.aps .org/doi/10.1103/ PhysRevLett.50.761

[115] Fauster T, Himpsel F J, Fischer J E and Plummer E W 1983 Physical Review Letters 51 430-433 URL https://link.aps.org/doi/10.1103/PhysRevLett. 51.430

[116] Harris C B, Ge N H, Lingle R L, McNeill J D and Wong C M 1997 Annual Review of Physical Chemistry 48 711-744 URL https://doi.org/10.1146/annurev. physchem.48.1.711

[117] Echenique P M and Pendry J B 1978 Journal of Physics C: Solid State Physics 11 2065-2075 URL http://dx.doi.org/10.1088/0022-3719/11/10/017

[118] Smith N V 1985 Physical Review B 32 3549-3555 URL https://link.aps.org/ doi/10.1103/PhysRevB.32.3549

[119] Smith N V and Woodruff D P 1986 Progress in Surface Science 21 295-370 URL http://www.sciencedirect.com/science/article/pii/0079681686900043

[120] Giesen K, Hage F, Himpsel F J, Riess H J and Steinmann W 1986 Physical Review B 33 5241-5244 URL https://link.aps.org/doi/10.1103/PhysRevB.33.5241

[121] Höfer U, Shumay I L, Reuß C, Thomann U, Wallauer W and Fauster T 1997 Science 2771480 URL http://science.sciencemag.org/content/277/5331/ 1480 . abstract

[122] Preobrajenski A B, Vinogradov A S and Mårtensson N 2004 Physical Review B 70 165404- URL https://link.aps.org/doi/10.1103/PhysRevB.70.165404

[123] Tonkikh A A, Voloshina E N, Werner P, Blumtritt H, Senkovskiy B, Güntherodt G, Parkin S S P and Dedkov Y S 2016 Scientific Reports 623547 EP - URL https://doi.org/10.1038/srep23547

[124] Laskowski R, Gallauner T, Blaha P and Schwarz K 2009 Journal of Physics: Condensed Matter 21104210 URL http://dx.doi.org/10.1088/0953-8984/ $21 / 10 / 104210$

[125] Preobrajenski A B, Vinogradov A S and Mårtensson N 2005 Surface Science 582 21-30 URL http://www.sciencedirect.com/science/article/ pii/S0039602805002426

[126] Fischer N, Schuppler S, Fauster T and Steinmann W 1990 Physical Review B 42 9717-9719 URL https://link.aps.org/doi/10.1103/PhysRevB. 42.9717 
[127] Passek F and Donath M 1992 Physical Review Letters 69 1101-1104 URL https://link.aps.org/doi/10.1103/PhysRevLett.69.1101

[128] Laturia A, Van de Put M L and Vandenberghe W G 2018 npj 2D Materials and Applications 26 URL https://doi.org/10.1038/s41699-018-0050-x

[129] Prakash S and Joshi S K 1970 Physical Review B 2 915-927 URL https: //link.aps.org/doi/10.1103/PhysRevB.2.915

[130] Claessen R, Carstensen H and Skibowski M 1988 Physical Review B $3812582-$ 12588 URL https://link.aps.org/doi/10.1103/PhysRevB. 38.12582

[131] Zhu X Y 2004 Surface Science Reports 56 1-83 URL http://www. sciencedirect.com/science/article/pii/S0167572904000779

[132] Muntwiler M, Yang Q and Zhu X Y 2009 Journal of Electron Spectroscopy and Related Phenomena 174 116-124 URL http://www.sciencedirect.com/ science/article/pii/S0368204809000577

[133] Prada E, Alvarez J V, Narasimha-Acharya K L, Bailen F J and Palacios J J 2015 Physical Review B 91 245421-URL https://link.aps.org/doi/10.1103/ PhysRevB.91.245421

[134] Link S, Sievers J, Dürr H A and Eberhardt W 2001 Journal of Electron Spectroscopy and Related Phenomena 114-116 351-355 URL http://www. sciencedirect.com/science/article/pii/S0368204800002462

[135] Stoner E C and Whiddington R 1936 Proceedings of the Royal Society of London. Series A - Mathematical and Physical Sciences 154 656-678 URL https://doi. org/10.1098/rspa.1936.0075

[136] Stoner E C 1939 Proceedings of the Royal Society of London. Series A. Mathematical and Physical Sciences 169 339-371 URL https://doi.org/10. 1098/rspa.1939.0003

[137] Greber T, Kreutz T J and Osterwalder J 1997 Physical Review Letters 79 44654468 URL https://link.aps.org/doi/10.1103/PhysRevLett.79.4465

[138] Kreutz T J, Greber T, Aebi P and Osterwalder J 1998 Physical Review B 58 1300-1317 URL https://link.aps.org/doi/10.1103/PhysRevB.58.1300

[139] Thomann U, Reuß C, Fauster T, Passek F and Donath M 2000 Physical Review B 61 16163-16167 URL https://link.aps.org/doi/10.1103/PhysRevB.61. 16163

[140] Schmidt A B, Pickel M, Donath M and Weinelt M 2007 Journal of Magnetism and Magnetic Materials 310 2330-2332 URL http://www.sciencedirect.com/ science/article/pii/S0304885306024000

[141] Weinelt M, Schmidt A B, Pickel M and Donath M 2007 Progress in Surface Science 82 388-406 URL http://www.sciencedirect.com/science/article/ $\mathrm{pii/S0079681607000226}$ 
[142] Paniago R, Matzdorf R, Meister G and Goldmann A 1995 Surface Science 336 113-122 URL http://www.sciencedirect.com/science/article/pii/ 0039602895005099

[143] Kouvel J S and Comly J B 1968 Physical Review Letters 20 1237-1239 URL https://link.aps.org/doi/10.1103/PhysRevLett.20.1237

[144] Hengsberger M, Muntwiler M, Lobo-Checa J and Greber T 2006 Source for spin-polarized electrons patent, filed July 2006, published on January 18, 2007 by the World Intellectual Property Organization, Int. Publication Number WO 2007/006168 URL https://www.wipo.int/portal/en/index.html

[145] Beaurepaire E, Merle J C, Daunois A and Bigot J Y 1996 Physical Review Letters 76 4250-4253 URL https: //link. aps.org/doi/10.1103/PhysRevLett. 76.4250

[146] Cinchetti M, Sánchez Albaneda M, Hoffmann D, Roth T, Wüstenberg J P, Krauß M, Andreyev O, Schneider H C, Bauer M and Aeschlimann M 2006 Physical Review Letters 97 177201-URL https://link.aps.org/doi/10.1103/ PhysRevLett .97.177201

[147] Rhie H S, Dürr H A and Eberhardt W 2003 Physical Review Letters $90247201-$ URL https://link.aps.org/doi/10.1103/PhysRevLett.90.247201

[148] Bovensiepen U 2007 Journal of Physics: Condensed Matter 19083201 URL http://dx.doi.org/10.1088/0953-8984/19/8/083201

[149] Koopmans B, Malinowski G, Dalla Longa F, Steiauf D, Fähnle M, Roth T, Cinchetti M and Aeschlimann M 2010 Nature Materials 9 259-265 URL https: //doi.org/10.1038/nmat2593

[150] Johannsen J C, Ulstrup S, Cilento F, Crepaldi A, Zacchigna M, Cacho C, Turcu I C E, Springate E, Fromm F, Raidel C, Seyller T, Parmigiani F, Grioni M and Hofmann P 2013 Physical Review Letters 111 027403- URL https://link.aps. org/doi/10.1103/PhysRevLett.111.027403

[151] Gierz I, Petersen J C, Mitrano M, Cacho C, Turcu I C E, Springate E, Stöhr A, Köhler A, Starke U and Cavalleri A 2013 Nature Materials 121119 EP - URL https://doi.org/10.1038/nmat3757

[152] Gierz I, Calegari F, Aeschlimann S, Chávez Cervantes M, Cacho C, Chapman R T, Springate E, Link S, Starke U, Ast C R and Cavalleri A 2015 Physical Review Letters 115 086803-URL https://link.aps.org/doi/10.1103/PhysRevLett. 115.086803

[153] Ulstrup S, Johannsen J C, Cilento F, Miwa J A, Crepaldi A, Zacchigna M, Cacho C, Chapman R, Springate E, Mammadov S, Fromm F, Raidel C, Seyller T, Parmigiani F, Grioni M, King P D C and Hofmann P 2014 Physical Review Letters 112 257401-URL https://link.aps.org/doi/10.1103/PhysRevLett. 112.257401 
[154] Gierz I and Cavalleri A 2016 Structural Dynamics 3051301 URL https://doi. org/10.1063/1.4964777

[155] Kaloni T P, Cheng Y C and Schwingenschlögl U 2012 Journal of Materials Chemistry 22 919-922 URL http://dx.doi.org/10.1039/C1JM14895H

[156] Gierz I 2014 Nature Physics 1112 EP - URL https://doi.org/10.1038/ nphys3206

[157] Winzer T and Malić E 2012 Physical Review B 85 241404- URL https://link. aps.org/doi/10.1103/PhysRevB.85.241404

[158] Plötzing T, Winzer T, Malic E, Neumaier D, Knorr A and Kurz H 2014 Nano Letters 14 5371-5375 URL https://doi.org/10.1021/n1502114w

[159] Roth S, Matsui F, Greber T and Osterwalder J 2013 Nano Letters 13 2668-2675 URL https ://doi .org/10.1021/nl400815w

[160] Roth S, Greber T and Osterwalder J 2016 ACS Nano 10 11187-11195 URL https://doi.org/10.1021/acsnano.6b06240

[161] Wang E, Lu X, Ding S, Yao W, Yan M, Wan G, Deng K, Wang S, Chen G, Ma L, Jung J, Fedorov A V, Zhang Y, Zhang G and Zhou S 2016 Nature Physics 12 1111 EP - URL https://doi.org/10.1038/nphys3856

[162] Wang E, Chen G, Wan G, Lu X, Chen C, Avila J, Fedorov A V, Zhang G, Asensio M C, Zhang Y and Zhou S 2016 Journal of Physics: Condensed Matter 28444002 URL http://dx.doi.org/10.1088/0953-8984/28/44/444002

[163] Joucken F, Quezada-López E A, Avila J, Chen C, Davenport J L, Chen H, Watanabe K, Taniguchi T, Asensio M C and Velasco J 2019 Physical Review B 99 161406-URL https://link.aps.org/doi/10.1103/PhysRevB.99.161406

[164] Hengsberger M, Roth S and Schuler A 2019 Vuv two-photon photoemission from h-bn/ni(111): the $\pi^{\star}$ band at $\overline{\mathrm{k}}$ unpublished 